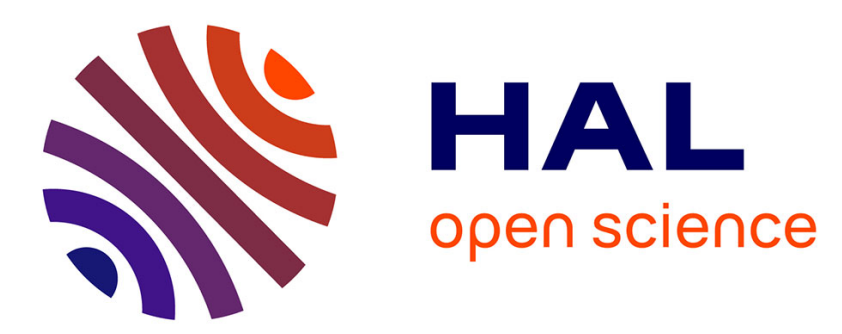

\title{
Gamma Rays from Type Ia Supernova SN 2014J
}

E Churazov, Rachid Sunyaev, Jordi Isern, I. Bikmaev, E. Bravo, N. Chugai, S.

Grebenev, Pierre Jean, Jürgen Knödlseder, François Lebrun, et al.

\section{To cite this version:}

E Churazov, Rachid Sunyaev, Jordi Isern, I. Bikmaev, E. Bravo, et al.. Gamma Rays from Type Ia Supernova SN 2014J. The Astrophysical Journal, 2015, 812 (1), pp.62. 10.1088/0004-637X/812/1/62 . hal-01381944

\section{HAL Id: hal-01381944 \\ https://hal.science/hal-01381944}

Submitted on 14 Oct 2016

HAL is a multi-disciplinary open access archive for the deposit and dissemination of scientific research documents, whether they are published or not. The documents may come from teaching and research institutions in France or abroad, or from public or private research centers.
L'archive ouverte pluridisciplinaire HAL, est destinée au dépôt et à la diffusion de documents scientifiques de niveau recherche, publiés ou non, émanant des établissements d'enseignement et de recherche français ou étrangers, des laboratoires publics ou privés. 


\title{
GAMMA RAYS FROM TYPE Ia SUPERNOVA SN 2014J
}

\author{
E. Churazov ${ }^{1,2}$, R. Sunyaev ${ }^{1,2}$, J. Isern ${ }^{3}$, I. Bikmaev ${ }^{4,5}$, E. Bravo ${ }^{6}$, N. Chugai ${ }^{7}$, S. Grebenev ${ }^{1}$, \\ P JeAN $^{8,9}$, J. KNÖDlSEDER ${ }^{8,9}$, F. Lebrun ${ }^{10}$, and E. KuUlKers ${ }^{11}$ \\ ${ }^{1}$ Space Research Institute (IKI), Profsouznaya 84/32, Moscow 117997, Russia \\ 2 Max Planck Institute for Astrophysics, Karl-Schwarzschild-Strasse 1, D-85741 Garching, Germany \\ ${ }^{3}$ Institut for Space Sciences (ICE-CSIC/IEEC), E-08193 Bellaterra, Spain \\ ${ }^{4}$ Kazan Federal University (KFU), Kremlevskaya Strasse, 18, Kazan, Russia \\ ${ }^{5}$ Academy of Sciences of Tatarstan, Bauman Strasse, 20, Kazan, Russia \\ ${ }^{6}$ E.T.S.A.V., Univ. Politecnica de Catalunya, Carrer Pere Serra 1-15, E-08173 Sant Cugat del Valles, Spain \\ ${ }^{7}$ Institute of Astronomy of the Russian Academy of Sciences, 48 Pyatnitskaya Street, 119017, Moscow, Russia \\ ${ }^{8}$ Université de Toulouse; UPS-OMP; IRAP; Toulouse, France \\ ${ }^{9}$ CNRS; IRAP; 9 Av. colonel Roche, BP 44346, F-31028 Toulouse cedex 4, France \\ ${ }^{10}$ APC, Univ Paris Diderot, CNRS/IN2P3, CEA/Irfu, Obs de Paris, Sorbonne Paris Cité, France \\ ${ }^{11}$ European Space Astronomy Centre (ESA/ESAC), Science Operations Department, \\ P.O. Box 78, E-28691 Villanueva de la Cañada, Madrid, Spain \\ Received 2015 January 30; accepted 2015 September 4; published 2015 October 8
}

\begin{abstract}
The whole set of INTEGRAL observations of Type Ia supernova SN 2014J, covering the period 19-162 days after the explosion, has been analyzed. For spectral fitting the data are split into early and late periods covering days $19-35$ and $50-162$, respectively, optimized for ${ }^{56} \mathrm{Ni}$ and ${ }^{56} \mathrm{Co}$ lines. As expected for the early period, much of the gamma-ray signal is confined to energies below $\sim 200 \mathrm{keV}$, while for the late period it is strongest above $400 \mathrm{keV}$. In particular, in the late period, ${ }^{56} \mathrm{Co}$ lines at 847 and $1248 \mathrm{keV}$ are detected at $4.7 \sigma$ and $4.3 \sigma$, respectively. The light curves in several representative energy bands are calculated for the entire period. The resulting spectra and light curves are compared with a subset of models. We confirm our previous finding that the gamma-ray data are broadly consistent with the expectations for canonical one-dimensional models, such as delayed detonation or deflagration models for a near-Chandrasekhar mass white dwarf. Late optical spectra (day 136 after the explosion) show rather symmetric Co and Fe line profiles, suggesting that, unless the viewing angle is special, the distribution of radioactive elements is symmetric in the ejecta.
\end{abstract}

Key words: gamma rays: general - methods: statistical - nuclear reactions, nucleosynthesis, abundances radiative transfer - supernovae: general - supernovae: individual (SN 2014J)

\section{INTRODUCTION}

A Type Ia supernova is believed to be a thermonuclear explosion of a carbon-oxygen (CO) white dwarf (Hoyle \& Fowler 1960) in a binary system (see, e.g., Hillebrandt \& Niemeyer 2000; Imshennik \& Dunina-Barkovskaya 2005, for a review). Most popular scenarios of the explosion include (i) a gradual increase of the mass toward the Chandrasekhar limit (e.g., Whelan \& Iben 1973), (ii) a merger/collision of two WDs (e.g., Iben \& Tutukov 1984; Webbink 1984; Kushnir et al. 2013), and (iii) an initial explosion at the surface of the sub-Chandrasekhar WD, which triggers a subsequent explosion of the bulk of the material (e.g., Nomoto \& Sugimoto 1977; Hoeflich \& Khokhlov 1996). In all of the scenarios, a thermonuclear runaway converts a substantial fraction of $\mathrm{CO}$ mass into iron-group elements and the energy released powers the explosion itself. The optical light of the supernova (SN) is in turn powered by the decay of radioactive elements, synthesized during the explosion. For the first year after the explosion the decay chain ${ }^{56} \mathrm{Ni} \rightarrow{ }^{56} \mathrm{Co} \rightarrow{ }^{56} \mathrm{Fe}$ is of prime importance. As long as the expanding ejecta are optically thick for gamma rays, the bulk of the decay energy is thermalized and is re-emitted in the UV, optical, and IR bands. After several tens of days, the ejecta become optically thin for gamma rays, making the Type Ia SN (SN Ia) a powerful source of gamma photons.

Here we report the results of INTEGRAL observations of SN 2014J covering a period from $\sim 16$ to $\sim 162$ days after the explosion.
The analysis of the SN 2014J data obtained by INTEGRAL has been reported in Churazov et al. (2014b; days $\sim 50-100$ since explosion), Diehl et al. (2014; days 16-19), and Isern et al. (2015; days 16-35); see also Diehl et al. (2015). Despite its proximity, SN 2014J is an extremely faint source in gamma rays and the expected signal is below $1 \%$ of the background. This makes the results sensitive to the procedure adopted to handle the background by different groups and has led to tension between some results. Here we have combined all INTEGRAL data and uniformly processed them using the same procedure as in Churazov et al. (2014b). The resulting spectra and light curves are compared with the predictions of basic Type Ia models.

Current state-of-the-art three-dimensional (3D) simulations of Type Ia explosions (e.g., Seitenzahl et al. 2013; Fink et al. 2014; Moll et al. 2014) lead to a complicated distribution of burning products in the ejecta and introduce a viewing angle dependence in the predicted gamma-ray flux. However, the overall significance of the SN 2014J detection in gamma rays by INTEGRAL (see Sections 3 and 5) corresponds to $\sim 10$ standard deviations. This precludes a very detailed modelindependent analysis. We therefore took a conservative approach of comparing the data with a subset of popular onedimensional (1D) SN Ia models (see Section 4), some of which were used in Milne et al. (2004) for assesment of SN Ia gamma-ray codes. While these models do not describe the full complexity of SN Ia ejecta, they can serve as useful indicators of the most basic characteristics of the explosion, including the 
total mass of radioactive nickel, total mass of the ejecta and the expansion velocity. We also verify (Section 5.3) whether adding an extra component, corresponding to a transparent clump of radioactive $\mathrm{Ni}$, on top of the best-fitting 1D model significantly improves the fit. In Section 6 we make several basic consistency checks of gamma-ray and optical data, using optical observations taken quasi-simultaneously with INTEGRAL observations. Section 7 provides the summary of our results.

\section{SN 2014J IN M82}

SN 2014J in M82 was discovered (Fossey et al. 2014) on 2014 January 21. The reconstructed (Zheng et al. 2014; Goobar et al. 2015) date of the explosion is January 14.75 UT with an uncertainty of the order of \pm 0.3 days. At the distance of M82 $(\sim 3.5 \mathrm{Mpc})$, this is the nearest SN Ia in several decades. The proximity of SN 2014J triggered many follow-up observations, including those by INTEGRAL (Kuulkers 2014).

The $\mathrm{SN}$ is located $\sim 1 \mathrm{kpc}$ from the nucleus of M82 and has a strong $\left(A_{V} \sim 2\right)$ and complicated absorption in the UV-optical band (e.g., Amanullah et al. 2014; Foley et al. 2014; Goobar et al. 2014; Kawabata et al. 2014; Welty et al. 2014; Brown et al. 2015; Marion et al. 2015; Patat et al. 2015).

From the light curves and spectra SN 2014J appears to be a "normal" SN Ia with no large mixing (e.g., Ashall et al. 2014; Marion et al. 2015), consistent with the delayed-detonation (DD) models. Detection of stable Ni (Friesen et al. 2014; Telesco et al. 2015) in IR suggests a high density of burning material (see, e.g., Shigeyama et al. 1992), characteristic of near-Chandrasekhar WDs.

Searches in X-ray, radio, and optical bands (including preSN observations of M82) did not reveal any evidence for accretion onto the WD before the explosion, any candidate for a companion star, or compelling evidence for a large amount of circumbinary material, implicitly supporting the DD scenario (Kelly et al. 2014; Margutti et al. 2014; Nielsen et al. 2014; Pérez-Torres et al. 2014), although some single-degenerate scenarios are not excluded.

In gamma rays, the first detection of SN 2014J in ${ }^{56}$ Co lines was reported about 50 days after the explosion (Churazov et al. 2014a). The gamma-ray signal from SN 2014J was also reported in the earlier phase $\sim 16-35$ days after the explosion (Diehl et al. 2014; Isern et al. 2014).

Throughout this paper we adopt the distance to M82 (and to SN 2014J) as $3.5 \mathrm{Mpc}$. The recent analysis by Foley et al. (2014) suggests a distance of $3.27 \pm 0.2 \mathrm{Mpc}$. This estimate is formally consistent with $D \sim 3.53 \pm 0.26 \mathrm{Mpc}$ from Karachentsev \& Kashibadze (2006) and our adopted value. Nevertheless, one should bear in mind that all fluxes and normalizations of best-fitting models can be overestimated (or underestimated) by as much as $\sim 20 \%$.

The only other SN sufficiently bright to allow for detailed study in gamma rays from ${ }^{56} \mathrm{Ni}$ and ${ }^{56} \mathrm{Co}$ decay is the Type II SN 1987A in the Large Magellanic Cloud. In SN 1987A the down-scattered hard X-ray continuum was first seen half a year after the explosion (Dotani et al. 1987; Sunyaev et al. 1987, 1990), while $\gamma$-ray lines of ${ }^{56}$ Co were detected several months later (Matz et al. 1988; Teegarden et al. 1989). While SN 2014J is more than 60 times further away from us than SN 1987A, the larger amount of radioactive ${ }^{56} \mathrm{Ni}$ and less massive/opaque ejecta in $\mathrm{SNe}$ Ia have made the detection of gamma rays from SN 2014J possible.

\section{INTEGRAL OBSERVATIONS AND BASIC DATA ANALYSIS}

INTEGRAL is an ESA scientific mission dedicated to fine spectroscopy and imaging of celestial $\gamma$-ray sources in the energy range $15 \mathrm{keV}-10 \mathrm{MeV}$ (Winkler et al. 2003).

The INTEGRAL data used here were accumulated during revolutions 1380-1386, 1391-1407, and 1419-1428 ${ }^{12}$, corresponding to the period $\sim 16-162$ days after the explosion.

In the analysis, we follow the procedures described in Churazov et al. (2014b) and Isern et al. (2013) and use the data of two instruments SPI and ISGRI/IBIS on board INTEGRAL.

\subsection{SPI}

SPI is a coded-mask germanium spectrometer on board INTEGRAL. The instrument consists of 19 individual $\mathrm{Ge}$ detectors, has a field of view of $\sim 30^{\circ}$ (at zero response), an effective area $\sim 70 \mathrm{~cm}^{2}$ at $0.5 \mathrm{MeV}$, and energy resolution of $\sim 2 \mathrm{keV}$ (Roques et al. 2003; Vedrenne et al. 2003). The effective angular resolution of SPI is $\sim 2^{\circ}$. During SN 2014J observations 15 out of 19 detectors were operating, resulting in slightly reduced sensitivity and imaging capabilities compared to the initial configuration.

Periods of very high and variable background due to solar flares and passage through radiation belts were omitted from the analysis. In particular, based on the SPI anticoincidence system count-rates, the revolutions 1389 and 1390 were completely excluded, as well as parts of revolutions 1405, $1406,1419,1423$, and 1426. The data analysis follows the scheme implemented for the analysis of the Galactic Center positron annihilation emission (Churazov et al. 2005, 2011). We used only "single" events (Vedrenne et al. 2003), and for each detector a linear relation between the energy and the channel number was assumed and calibrated (separately for each orbit), using the observed energies of background lines at 198, 438, 584, 882, 1764, 1779, 2223, and $2754 \mathrm{keV}$.

The flux of the SN $S(E)$ at energy $E$ and the background rates in individual detectors $B_{i}(E, t)$ were derived from a simple model of the observed rates $D_{i}(E, t)$ in individual SPI detectors, where $i$ is the detector number and $t$ is the time of observation with a typical exposure of $2000 \mathrm{~s}$ :

$$
D_{i}(E, t) \approx S(E) \times R_{i}(E, t)+B_{i}(E, t) .
$$

Here $R_{i}(E, t)$ is the effective area of the $i$ th detector, as seen from the source position in a given observation. The background rate is assumed to be linearly proportional to the Ge detectors' saturated event rate $G_{\text {Sat }}(t)$ above $8 \mathrm{MeV}$, averaged over all detectors, i.e., $B_{i}(E, t)=\beta_{i}(E) G_{\text {Sat }}(t)+$ $C_{i}(E)$, where $C_{i}(E)$ does not depend on time. The coefficients $S(E), \beta_{i}(E)$, and $C_{i}(E)$ are free parameters of the model and are obtained by minimizing $\chi^{2}$ for the entire data set. Even though the number of counts in individual exposures is low, it is still possible to use a plain $\chi^{2}$ approach as long as the errors are estimated using the mean count rate and the total number of counts in the entire data set is large (Churazov et al. 1996). The linear nature of the model allows for straightforward estimation of statistical errors.

Despite its proximity, SN 2014J is still an extremely faint source in $\gamma$-rays. Figure 1 shows a comparison of the

\footnotetext{
${ }^{12}$ http://www.cosmos.esa.int/web/integral/schedule-information
} 


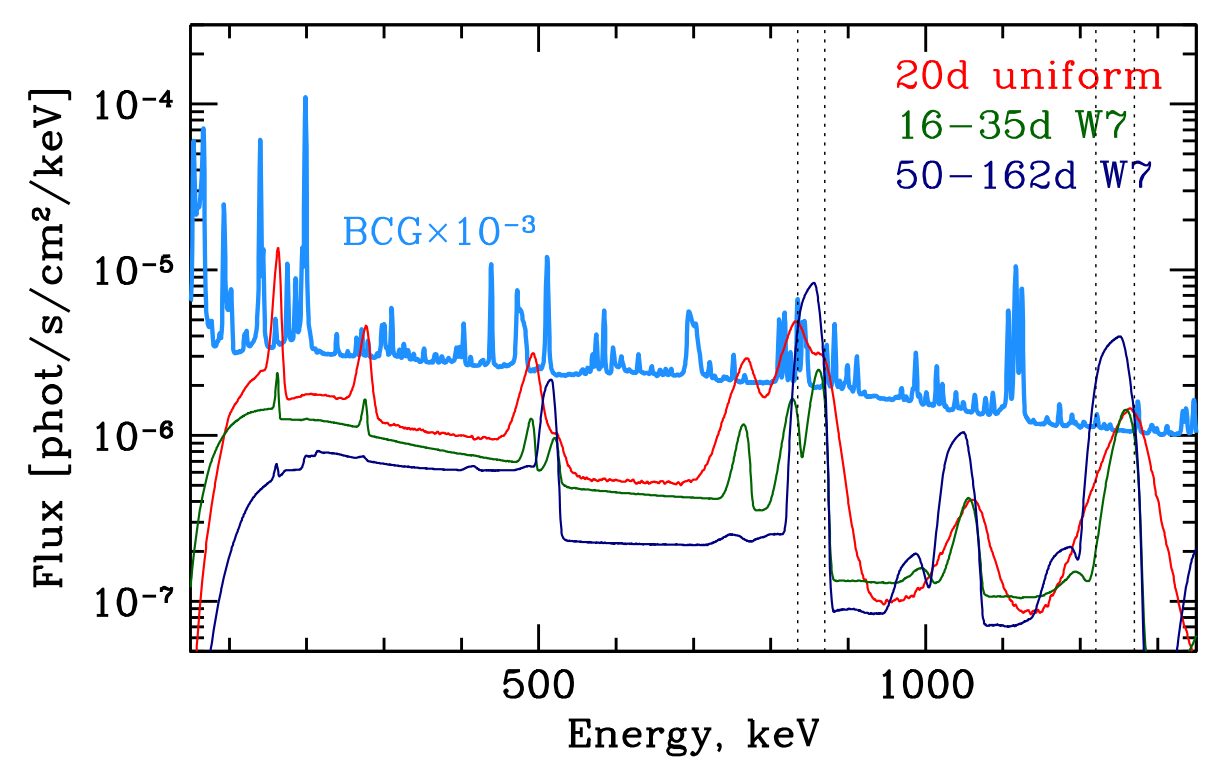

Figure 1. SPI quiescent background in comparison with the representative model spectra. SPI background is multiplied by a factor $10^{-3}$. Green and blue lines correspond to the $\mathrm{W} 7$ model (Nomoto et al. 1984) averaged over the early and late periods (see Section 3.3), respectively. The red line shows the 3PAR model from Churazov et al. (2014b) for day 20 after the explosion. In this model all elements, including radioactive isotopes, are mixed uniformly though the whole ejecta. The robust prediction of all plausible models is the presence of two ${ }^{56} \mathrm{Co}$ lines at 847 and $1238 \mathrm{keV}$ during the late phase. Vertical lines show two energy bands used for making images. The "cleanest" SPI background is near the $1238 \mathrm{keV}$ line, where no strong instrumental lines are present.

quiescent SPI background, scaled down by a factor of $10^{3}$ with a sample of representative models. Two models labeled " $20 \mathrm{~d}$ uniform" and " $16-35 \mathrm{~d}$ W7" show the models for the early period of SN 2014J observations. The former model is based on the best-fitting 3PAR model to the SN spectra recorded between 50 and 100 days after explosion (Churazov et al. 2014b), recalculated for day 20. The model assumes uniform mixing of all elements, including radioactive ${ }^{56} \mathrm{Ni}$, across the ejecta. This model at day 20 produces prominent ${ }^{56} \mathrm{Ni}$ lines near 158 and $812 \mathrm{keV}$. The latter model (W7, see Section 4) averaged over the period 16-35 days does not include mixing and it produces much fainter lines. Finally the "50-162 d W7" model corresponds to later observations. The most prominent features of this model are the ${ }^{56} \mathrm{Co}$ lines at 847 and $1238 \mathrm{keV}$. Among all these features the ${ }^{56} \mathrm{Co}$ line at $1238 \mathrm{keV}$ is located in the least complicated portion of the background spectrum.

The spectral redistribution matrix accounts for the instrumental line broadening estimated from the data accumulated during SN 2014J observations. We parameterize the energy resolution as a Gaussian with the energy-dependent width

$$
\sigma_{i} \approx 0.94\left(E_{\text {line }} / 500\right)^{0.115} \mathrm{keV} .
$$

Compared to our previous analysis we have amended the spectral redistribution matrix of SPI by including low-energy tails, which are associated with the interactions (Compton scattering) of incoming photons inside the detector and in the surrounding material. These photons are still registered as single events in the SPI data, but their energies are lower than the true incident energy. We used the results of a Monte-Carlo simulation of the SPI energy/imaging response (Sturner et al. 2003) and folded-in our procedure of spectrum reconstruction described above. For steep spectra, taking account of a lowenergy tail results in a modest $\sim 10 \%$ change in the spectrum normalization, while for the very hard SN 2014J spectrum it produces a low-energy tail that provides large contribution to

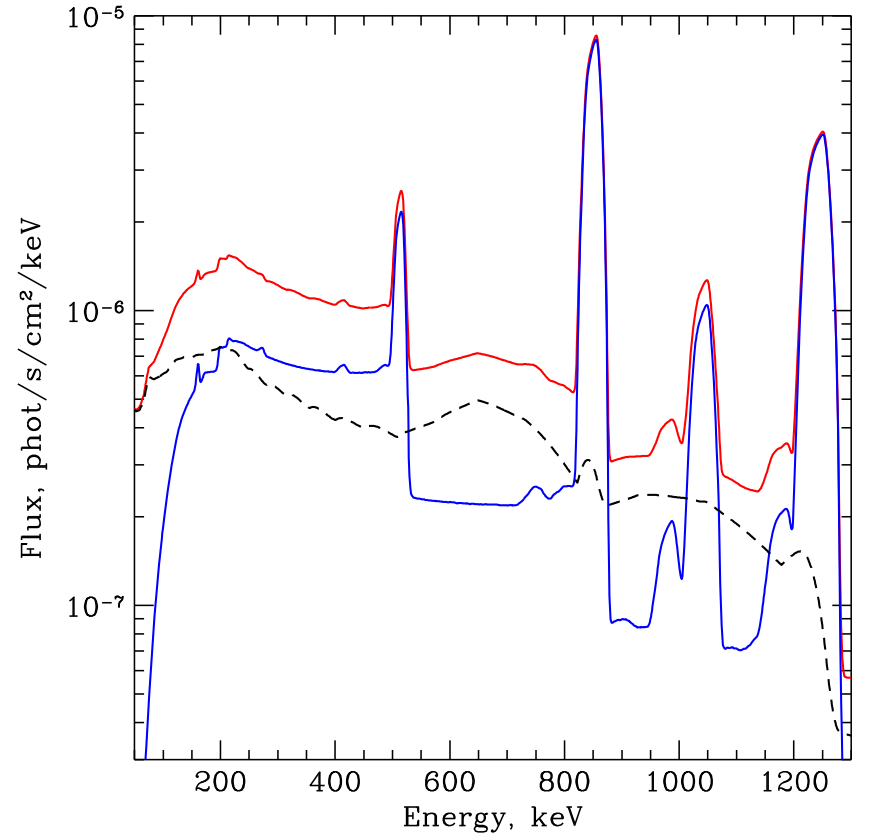

Figure 2. Estimated contribution of the off-diagonal terms in the SPI spectral response to the SN spectrum. The blue line shows the predicted spectrum of the W7 model for the late period, convolved with a simplified (nearly diagonal) SPI response. In this approximation the instrumental broadening is parameterized as an energy-dependent Gaussian with a width according to Equation (2). The red line shows the same spectrum convolved with the response that includes estimated off-diagonal terms, caused by Compton scattering of incident photons in the detector and surrounding structures. The off-diagonal component alone is shown with the dashed black line. The off-diagonal terms create a long lowenergy tail associated with gamma-ray lines. The impact on the brightest lines is small, while the continuum is strongly affected, especially at low energies. The model $\mathrm{W} 7$ is averaged over the period 50-162 days after the explosion.

the continuum, while fluxes of narrow lines remain unaffected (Figure 2). With this response matrix, the Crab Nebula spectrum, observed by INTEGRAL between 2014 February 21 and 23, is well described by a broken power law obtained by 
Jourdain \& Roques (2009) for earlier Crab Nebula observations with INTEGRAL.

In our analysis we usually ignore the part of the spectrum at energies higher than $1350 \mathrm{keV}$, since in the energy range between 1400 and $1700 \mathrm{keV}$ the instrument suffers from enhanced detector electronic noise, while at even higher energies only weaker lines from ${ }^{56}$ Co decay are expected (see Table 3 in Section 4.2). The convolution of the fiducial SN Ia model (see Section 4) with the simulated SPI response (Sturner et al. 2003) confirmed that the contribution of highenergy lines is negligible below $1350 \mathrm{keV}$, at least for "single" events considered here.

Inspection of Figure 1 shows that there is no chance to detect continuum in the SPI data for any of our fiducial models. For example, for a $100 \mathrm{keV}$ wide energy bin between 600 and $700 \mathrm{keV}$ the expected signal-to-noise ratio $(\mathrm{S} / \mathrm{N})$ after $4 \mathrm{~ms}$ observation between days 50 and 162 is $\sim 0.5 \sigma$. In the real data no evidence for significant continuum above $500 \mathrm{keV}$ was found in the time-averaged spectra (see Section 5.1 below). In Figure 2 the off-diagonal tail of the 847 and $1238 \mathrm{keV}$ lines dominates over intrinsic SN continuum, while the line shapes and fluxes are not affected.

In general, we consider the inclusion of the off-diagonal term in the response as an improvement compared to a pure diagonal response. We use this improved response throughout the paper, and at the same time in Section 5.1 we consider several data sets which include or exclude the SPI data below $\sim 400 \mathrm{keV}$. Inclusion of the low-energy $(\lesssim 400 \mathrm{keV})$ data boosts the $\mathrm{S} / \mathrm{N}$, while the exclusion of these data (dominated by off-diagonal continuum) makes spectral fits less prone to possible uncertainties in the off-diagonal term calibration.

To verify the whole SPI pipeline, we have made an independent analysis of the same data using the tools and procedures originally developed and tuned for SN 2011fe (see Isern et al. 2013). This analysis includes energy calibration, background modeling, and fitting of the background and source fluxes. Verification of these steps is important since the source (SN 2014J) is very faint and even subtle changes in the calibration might result in significant changes in the source spectrum. The fluxes in the $835-870 \mathrm{keV}$ band were derived using these two independent pipelines for every revolution during SN 2014J observations. Comparing fluxes point by point, we have found very good agreement, with the scatter well within statistical errors. The signal from SN 2014J is seen in both pipelines. No systematic trends of deviations with the variations of the flux level are found. We conclude that the results are fully consistent within the assumptions made on the background parameterization.

\subsection{ISGRI/IBIS}

The primary imaging instrument inboard INTEGRAL is IBIS (Ubertini et al. 2003) — a coded-mask aperture telescope with the CdTe-based detector ISGRI (Lebrun et al. 2003). It has higher sensitivity to continuum emission than SPI in the $20-300 \mathrm{keV}$ range ${ }^{13}$ and has a spatial resolution of $\sim 12^{\prime}$. We note here that neither ISGRI nor SPI can distinguish the emission of SN 2014J from the emission of any other source in M82. In particular, M82 hosts two ultra-luminous and variable sources (e.g., Bachetti et al. 2014; Sazonov et al. 2014) that contribute to the flux below $\sim 50 \mathrm{keV}$. ISGRI, however, can

\footnotetext{
13 http://www.cosmos.esa.int/web/integral/ao13
}

easily differentiate between M82 and M81, which are separated by $\sim 30^{\prime}$. The energy resolution of ISGRI is $\sim 10 \%$ at $100 \mathrm{keV}$. The ISGRI energy calibration uses the procedure implemented in OSA 10.039. The images in broad energy bands were reconstructed using a standard mask/detector cross-correlation procedure, tuned to produce zero signal on the sky if the count rate across the detector matches the pattern expected from pure background, which was derived from the same data set by stacking detector images. The noise in the resulting images is fully consistent with the expected level, determined by photon counting statistics. The fluxes in broad bands were calibrated using the Crab Nebula observations with INTEGRAL made between February 21 and 23. The model of Jourdain \& Roques (2009) was assumed as a reference.

\subsection{Light Curves, Spectra, and Images}

The light curves in several energy bands were generated using IGSRI and SPI data. The time bins ( $\sim 3$ days each) correspond to individual revolutions of the satellite. Finer time bins are not practical given that the source is very faint. The light curves are shown in Figures 3 and 4 together with a set of representative models (see Section 4). For the broad $100-200 \mathrm{keV}$ band the conversion of the ISGRI flux using the Crab spectrum as a reference is not very accurate because of the difference in the shape of the incident spectra. The conversion factor has been recalculated using several representative SN models, resulting in a modest $\sim 13 \%$ correction factor applied to the fluxes shown in Figure 3.

In principle, the spectra can be extracted for any interval covered by the observations, e.g., for individual revolutions, as is done above for the light curves in several broad bands. To compare the observed and predicted spectra we decided to split the data into two intervals covering 16-35 and 50-162 days after the explosion (see Table 1 and Figure 5). The gap between days 35 and 50 is partly due to a major solar flare. Below we refer to these two data sets as early and late periods.

Unlike the early period, when the emergence of ${ }^{56} \mathrm{Ni}$ lines depends strongly on the distribution of radioactive Ni through the ejecta, for the late period the emission in ${ }^{56} \mathrm{Co}$ lines is a generic prediction of all plausible models. Two energy bands optimal for detection of the SN signal in gamma rays are clear from Figure 1. These two bands, containing the most prominent ${ }^{56} \mathrm{Co}$ lines, were used to generate images. The images were extracted from SPI data from the late period as in Churazov et al. (2014b). That is, we vary the assumed position of the source and repeat the flux fitting procedure (see Section 3.1) for each position. The resulting images of the $\mathrm{S} / \mathrm{N}$ in the $835-870$ and $1220-1270 \mathrm{keV}$ energy bands are shown in Figure 6. In both energy bands the highest peaks $(4.7 \sigma$ and $4.3 \sigma$, respectively) coincide well (within 0.3 ) with the position of SN 2014J, marked by a cross.

The ISGRI spectra extracted at the known position of SN 2014J for the early and late periods are shown in Figure 7. The low-energy (less than $\sim 70 \mathrm{keV}$ ) part of the extracted spectrum is likely contaminated by other sources in M82.

\section{MODELS}

\subsection{A Set of Representative Models}

For comparison with the INTEGRAL data, we used a set of representative 1D models (Table 2) based on calculations of 


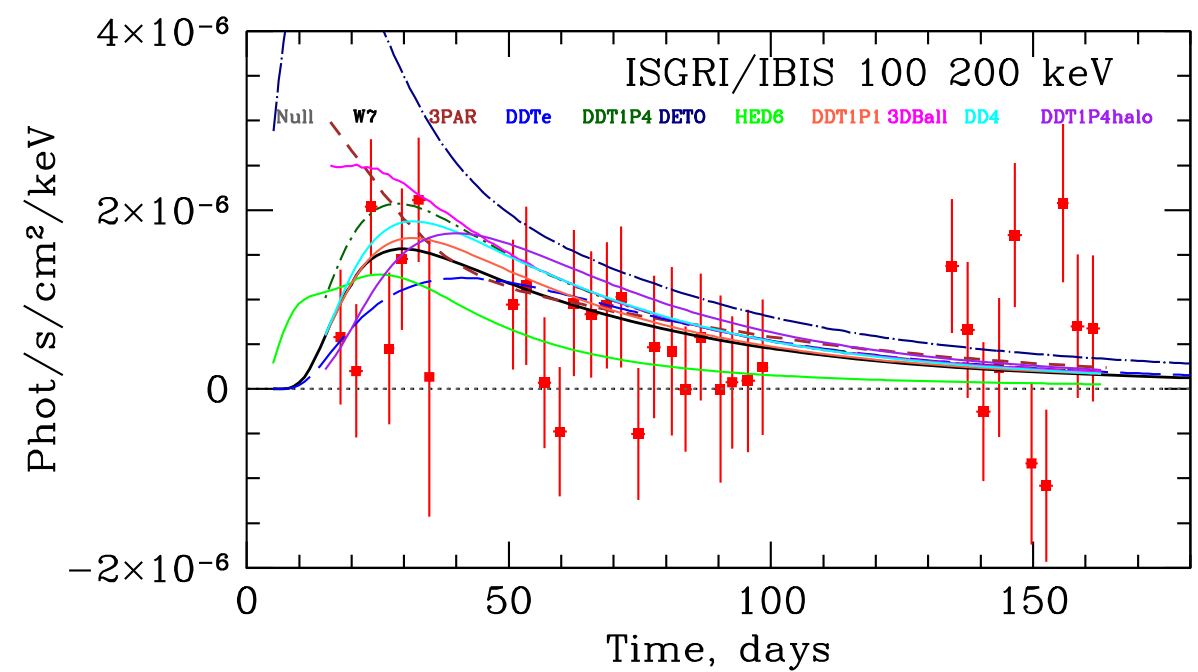

Figure 3. ISGRI light curve in the $100-200 \mathrm{keV}$ band. The $\mathrm{S} / \mathrm{N}$ in this band is expected to be the highest for the plausible models. The curves show the expected flux evolution for a set of models (see Section 4). Color coding is explained in the legend.

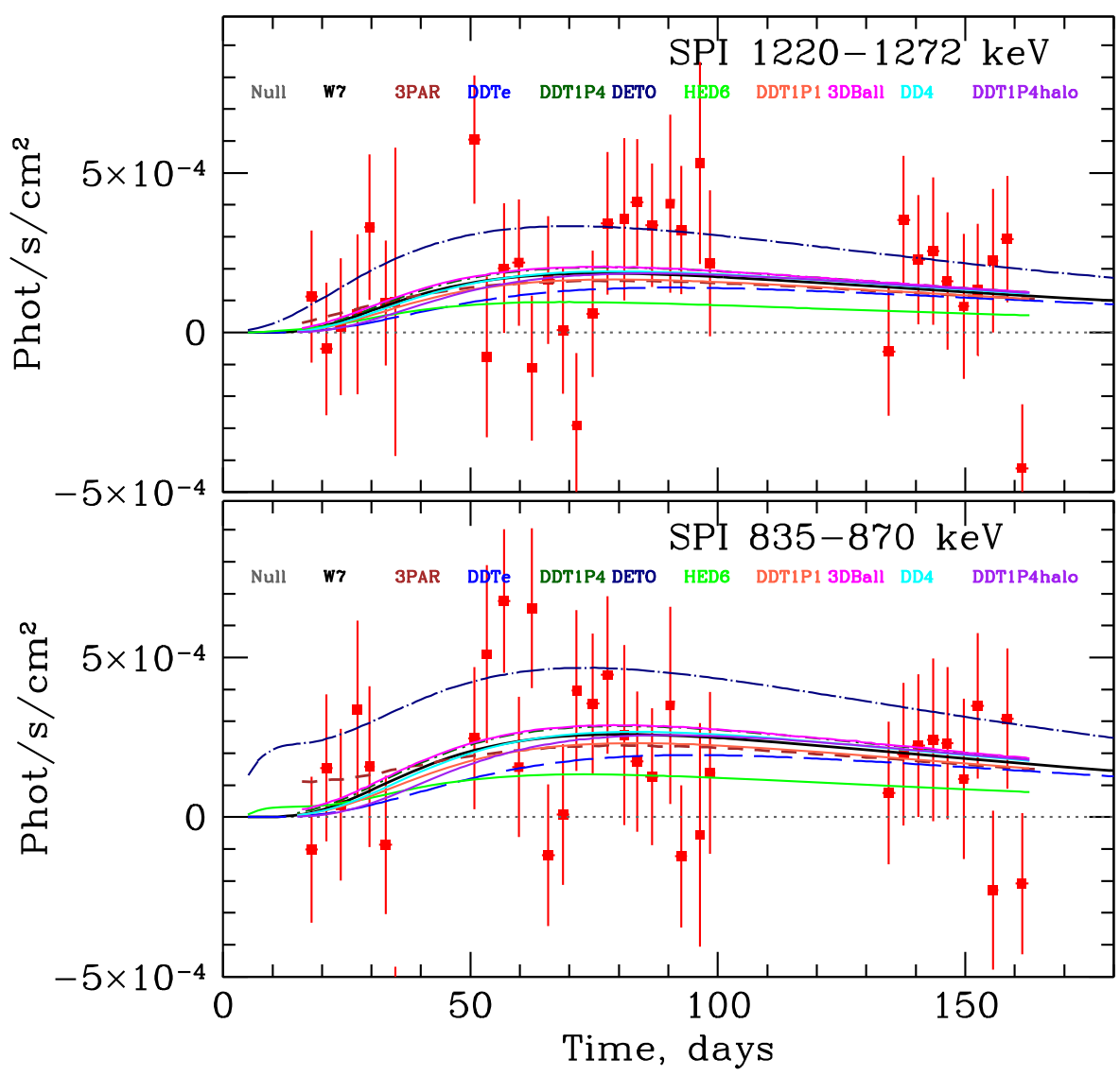

Figure 4. Same as in Figure 3 for SPI data in two narrow bands near the brightest ${ }^{56} \mathrm{Co}$ lines.

explosive nucleosynthesis models. To the first approximation, these models are characterized by the amount of radioactive nickel, the total mass of the ejecta, and the expansion velocity. Although current state-of-the-art simulations of Type Ia explosions can be done in 3D (e.g., Seitenzahl et al. 2013; Fink et al. 2014; Moll et al. 2014), using these models would introduce an additional viewing angle dependence. In order to avoid this extra degree of freedom, and given that the overall significance of the SN 2014J detection in gamma rays by INTEGRAL (see Sections 3 and 5) corresponds to only $\sim 10$ standard deviations, we decided to keep in this work only a set of 1D models to confront the data.

The set of models includes the deflagration model $W 7$ (Nomoto et al. 1984), pure detonation model DETO (Badenes 


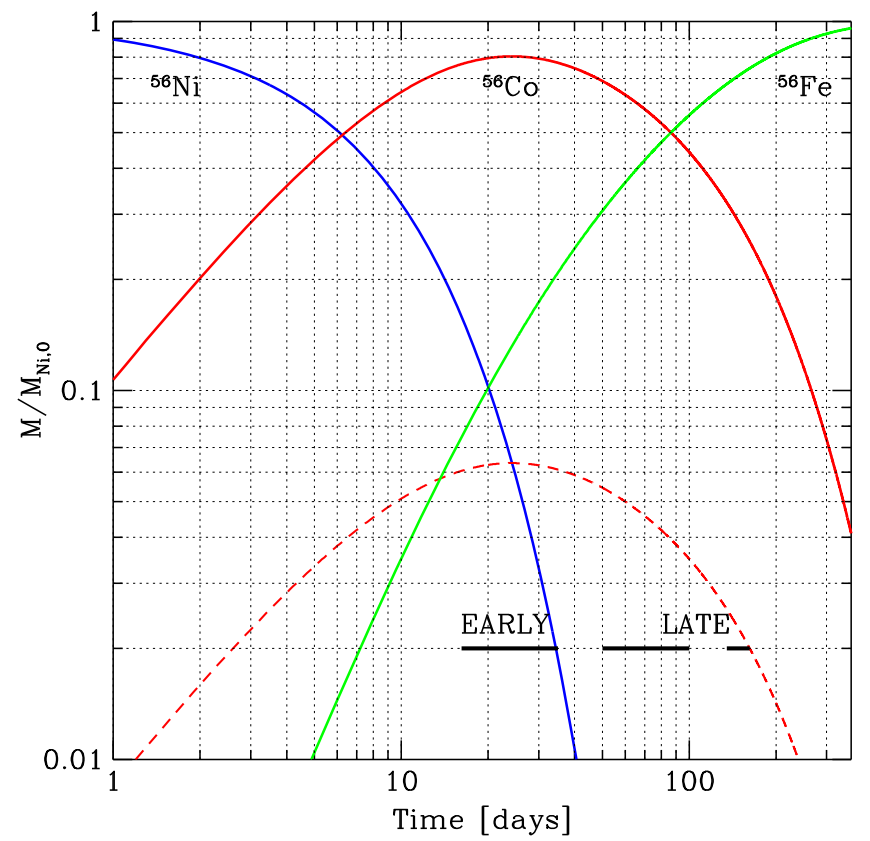

Figure 5. Early and late periods of INTEGRAL observations used for extraction of spectra, shown as thick horizontal bars. Three curves show the evolution of the ${ }^{56} \mathrm{Ni},{ }^{56} \mathrm{Co}$, and ${ }^{56} \mathrm{Fe}$ masses normalized to the initial ${ }^{56} \mathrm{Ni}$ mass. Note that opacity effects tend to suppress the emergence of gamma rays at early phases of the supernova evolution, unless radioactive isotopes are present in the outer layers of the ejecta, or the explosion is strongly asymmetric. The dashed red line shows the ${ }^{56} \mathrm{Co}$ mass scaled down by the ratio of $\mathrm{Co}$ and $\mathrm{Ni}$ decay times $\tau_{\mathrm{Co}} / \tau_{\mathrm{Ni}}$, which allows one to compare the expected relative strengths of $\mathrm{Ni}$ (blue curve) and $\mathrm{Co}$ (dashed red curve) gamma-ray lines as a function of time.

et al. 2003), the sub-Chandrasekhar model HED6 (Hoeflich \& Khokhlov 1996), and several variants of the delayed-detonation models: DD4 (Woosley \& Weaver 1991), DDTe (Badenes et al. 2003), DDT1p1, DDT1p4halo, ddt1p4, 3Dbbal (Isern et al. 2015). The ddt $1 \mathrm{p} 4$ model was built to match the mass of ${ }^{56} \mathrm{Ni}$ suggested by the early optical evolution of SN $2014 \mathrm{~J}$ as detected with the OMC of INTEGRAL (Isern et al. 2014; P. Hofflich 2015, private communication). In it, the transition density from deflagration to detonation was fixed at $1.4 \times 10^{7} \mathrm{~g} \mathrm{~cm}^{-3}$. Model DDT1p4halo is a variant of the latter in which the white dwarf is surrounded by a $0.2 M_{\odot}$ envelope, as might result from a delayed merger explosion. The $3 \mathrm{Dbbal}$ model is essentially the same as the ddt $1 \mathrm{p} 4$ plus a plume of $0.04 M_{\odot}$ of radioactive ${ }^{56} \mathrm{Ni}$ receding from the observer (see Isern et al. 2015 for details).

The emerging X-ray and gamma-ray radiation from the expanding SN Ia is determined by the total amount of radioactive isotopes, their distribution over velocities, the mass and the chemical composition of the ejecta and expansion rate. The processes are essentially the same as in SNe II (see, e.g., Sunyaev et al. 1987, for a prototypical example of a SN II-SN 1987A). However, the mass of the ejecta and expansion rate differ strongly, leading to a much earlier and stronger signal in gamma rays (see, e.g., Clayton et al. 1969; Woosley et al. 1981; Ambwani \& Sutherland 1988). A comprehensive set of computations of the expected gamma-ray flux for different representative models was presented in The \& Burrows (2014).

Here we use the results of similar calculations (see below), which account for line broadening, needed for systematic comparison with the INTEGRAL data.
A Monte-Carlo code follows the propagation of the $\gamma$ photons through the ejecta and accounts for scattering and photoabsorption of photons and annihilation of positrons. The predicted spectra were generated with a time step of one day, covering the entire observational period. These model spectra were then averaged over the periods of 16-35 and 50-162 days to provide fair comparison with the INTEGRAL results for the early and late periods, respectively. In particular, the effect of varying opacity in each model over the observational period is correctly captured by this procedure. The computations include full treatment of Compton scattering (coherent and incoherent), photoabsorption, and pair production (see Milne et al. 2004 for details). The positrons produced by $\beta^{+}$decay of ${ }^{56} \mathrm{Co}$ (19\% of all decays) annihilate in place via positronium formation. Both two-photon annihilation into the $511 \mathrm{keV}$ line and the orthopositronium continuum are included.

\subsection{Transparent Ejecta Model (TEM)}

As we discuss below (Section 5) the INTEGRAL data are broadly consistent with the subset of models listed in Table 2. However, Diehl et al. (2014) reported evidence of ${ }^{56} \mathrm{Ni}$ at the surface in the first observations of SN 2014J with INTEGRAL (see also Isern et al. 2015 for an alternative analysis of early SN $2014 \mathrm{~J}$ observations). The presence of radioactive material at the surface would be an important result, since the traditional models listed in Table 2 do not predict it. One can attempt to patch our 1D models with an additional component describing extra radioactive material at the surface. Assuming that the material at the surface is transparent to gamma rays, the fluxes of individual lines associated with $\mathrm{Ni}$ and $\mathrm{Co}$ decay, their energies and widths can be tied together. The transparency assumption is justified by the large velocities and small initial densities expected for matter at the surface of SNe ejecta. In any case, it provides a lower limit to the mass of radioactive material, as opacity would demand a larger gamma-ray production rate in order to explain a given gamma-ray flux. This approach allows us to describe many lines associated with a transparent clump using only three parameters. Below we refer to this component as a TEM, and use it in combination with the best-performing $\mathrm{W} 7$ model from our default set of 1D models (see Section 4.1), i.e., the data are compared with the predictions of the $\mathrm{W} 7+\mathrm{TEM}$ model. While this model by itself is not self-consistent, it can be used to answer the following questions.

1. Once the predicted signal for the $\mathrm{W} 7$ model is removed from the observed spectra, do the residuals resemble a signal expected from a transparent clump of radioactive material?

2. Given the statistics accumulated by INTEGRAL, how much radioactive material in a transparent clump can be "hidden" in the data on top of a given 1D model?

In this section we describe the TEM model and then apply it to the data in Section 5.3.

The TEM model assumes that all line energies are shifted proportionally to their energies (i.e., the same velocity structure for all lines), while their flux ratios follow the ratios predicted (Nadyozhin 1994) based on the decay chains of ${ }^{56} \mathrm{Ni} \rightarrow{ }^{56} \mathrm{Co}$ $\rightarrow{ }^{56} \mathrm{Fe}$ and ${ }^{57} \mathrm{Ni} \rightarrow{ }^{57} \mathrm{Co} \rightarrow{ }^{57} \mathrm{Fe}$. The list of the lines and their fluxes normalized to $1 M_{\odot}$ of ${ }^{56} \mathrm{Ni}$ are given in Table 3 . For a given time period the model has three parameters: the initial ${ }^{56} \mathrm{Ni}$ mass $\left(M_{\mathrm{Ni}}\right)$, the energy/redshift of the $847 \mathrm{keV}$ line $\left(E_{847}\right)$ 
Table 1

Data Sets

\begin{tabular}{lccc}
\hline \hline Set & Dates & Days Since Explosion & Exposure (ms) \\
\hline Early & 2014 Jan 31 to 2014 Feb 20 & 16 to 35 & 1.0 \\
Late & 2014 Mar 05 to 2014 Jun 25 & 50 to 162 & 4.3 \\
\hline
\end{tabular}

Note.

${ }^{\text {a }}$ Corrected for the periods of high background and the dead-time of SPI.
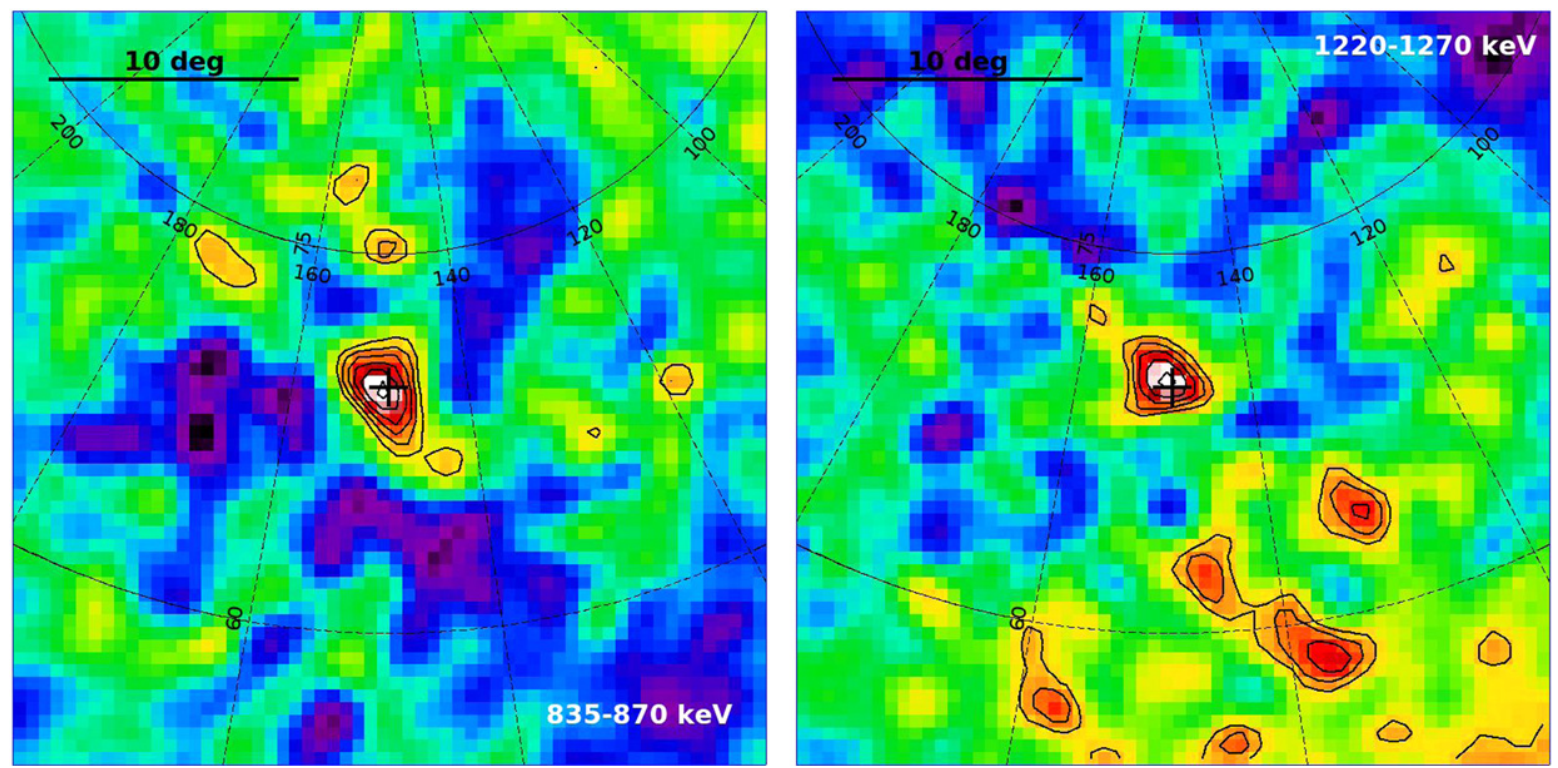

Figure 6. SPI images ( $/ \mathrm{N})$ during the late period in two narrow bands around the most prominent ${ }^{56}$ Co lines. Contours are at $2 \sigma, 2.5 \sigma, \ldots, 5 \sigma$. The cross shows the position of SN 2014J. The brightest peaks in each image coincide well with this position. Due to the dither pattern (http://www.cosmos.esa.int/web/integral) used during observations of SN 2014J the central part of the image is much better covered than the outer regions. It is therefore not surprising that the level of noise is increasing away from the nominal target.

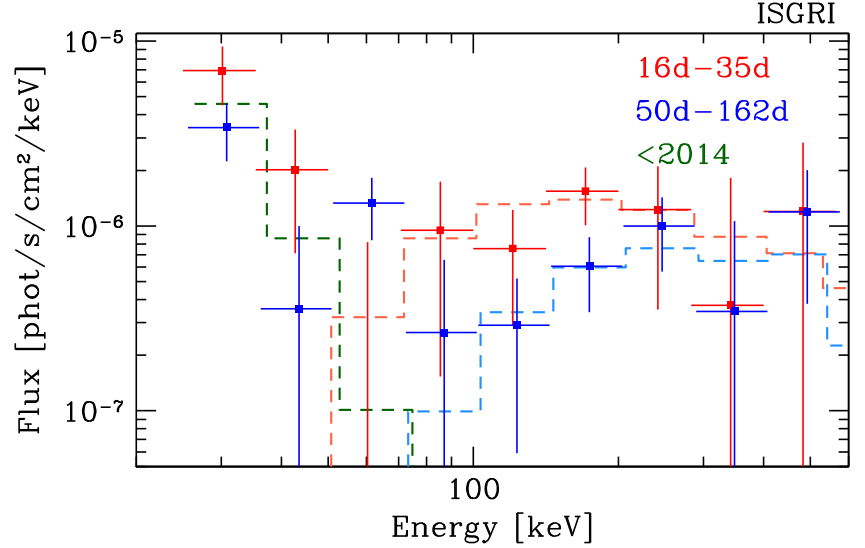

Figure 7. ISGRI spectrum measured at the position of SN 2014J during early (red) and late (blue) periods. The energies of the second set of points are multiplied by a factor 1.02 for the sake of clarity. Dashed histograms show the predicted spectra of the $\mathrm{W} 7 \mathrm{model}$ for the same periods. The agreement with the predictions is reasonable except for energies lower than $\sim 70 \mathrm{keV}$, where the spectrum is likely contaminated by other sources in M82 (see, e.g., Sazonov et al. 2014). The dark green line shows a crude approximation of the M82 spectrum measured before 2014 .

and the broadening of the $847 \mathrm{keV}$ line $\left(\sigma_{847}\right)$. The width of each line (Gaussian $\sigma$ ) is defined as

$$
\sigma_{\text {line }}=\sigma_{847} \times\left(\frac{E_{\text {line }}}{E_{847}}\right) \text {. }
$$

Table 2

Set of Models Used in the Paper

\begin{tabular}{lccc}
\hline \hline Model & $M_{\mathrm{Ni}}\left(M_{\odot}\right)$ & $M_{\text {tot }}\left(M_{\odot}\right)$ & $E_{K}\left(10^{51} \mathrm{erg}\right)$ \\
\hline DDT1p1 & 0.54 & 1.36 & 1.29 \\
DDT1p4halo & 0.62 & 1.55 & 1.3 \\
DDTe & 0.51 & 1.37 & 1.09 \\
DETO & 1.16 & 1.38 & 1.44 \\
HED6 & 0.26 & 0.77 & 0.72 \\
W7 & 0.59 & 1.38 & 1.24 \\
ddt1p4 & 0.66 & 1.36 & 1.35 \\
3Dbbal & $0.66+0.04^{\mathrm{a}}$ & 1.36 & 1.35 \\
DD4 & 0.61 & 1.39 & 1.24 \\
\hline
\end{tabular}

Note.

a Additional "plume" of ${ }^{56} \mathrm{Ni}$.

The ortho-positronium continuum and pair production by gamma-ray photons are neglected, while the $511 \mathrm{keV}$ line is added, assuming that $19 \%$ of ${ }^{56} \mathrm{Co}$ decays produce positrons, of which $25 \%$ form para-positronium yielding two $511 \mathrm{keV}$ photons.

\section{RESULTS}

\subsection{Combined ISGRI+SPI Spectrum}

The SPI images (Figure 6) for the late period unambiguously show the characteristic signatures of ${ }^{56}$ Co decay from SN 2014J. 
Table 3

Line Fluxes Averaged Over Days 50-162 for a Transparent Ejecta Model (TEM) for the Initial $1 M_{\odot}$ of ${ }^{56} \mathrm{Ni}$

\begin{tabular}{lccc}
\hline \hline$E_{\text {line }}(\mathrm{keV})$ & $F_{\text {line }} / F_{847}$ & Flux $^{\mathrm{a}}$ & Isotope \\
\hline 846.78 & 1.00 & $6.57 \times 10^{-4}$ & ${ }^{56} \mathrm{Co}$ \\
158.38 & $7.98 \times 10^{-3}$ & $5.25 \times 10^{-6}$ & ${ }^{56} \mathrm{Ni}$ \\
1561.80 & $1.12 \times 10^{-3}$ & $7.34 \times 10^{-7}$ & ${ }^{56} \mathrm{Ni}$ \\
749.95 & $3.99 \times 10^{-3}$ & $2.62 \times 10^{-6}$ & ${ }^{56} \mathrm{Ni}$ \\
269.50 & $2.87 \times 10^{-3}$ & $1.89 \times 10^{-6}$ & ${ }^{56} \mathrm{Ni}$ \\
480.44 & $2.87 \times 10^{-3}$ & $1.89 \times 10^{-6}$ & ${ }^{56} \mathrm{Ni}$ \\
811.85 & $6.86 \times 10^{-3}$ & $4.51 \times 10^{-6}$ & ${ }^{56} \mathrm{Ni}$ \\
511.00 & $9.50 \times 10^{-2}$ & $6.24 \times 10^{-5}$ & ${ }^{56} \mathrm{Co}$ \\
1037.83 & $1.40 \times 10^{-1}$ & $9.20 \times 10^{-5}$ & ${ }^{56} \mathrm{Co}$ \\
1238.28 & $6.80 \times 10^{-1}$ & $4.47 \times 10^{-4}$ & ${ }^{56} \mathrm{Co}$ \\
$1771.49^{\mathrm{b}}$ & $1.60 \times 10^{-1}$ & $1.05 \times 10^{-4}$ & ${ }^{56} \mathrm{Co}$ \\
$2034.92^{\mathrm{b}}$ & $7.90 \times 10^{-2}$ & $5.19 \times 10^{-5}$ & ${ }^{56} \mathrm{Co}$ \\
$2598.58^{\mathrm{b}}$ & $1.69 \times 10^{-1}$ & $1.11 \times 10^{-4}$ & ${ }^{56} \mathrm{Co}$ \\
$3253.60^{\mathrm{b}}$ & $7.40 \times 10^{-2}$ & $4.86 \times 10^{-5}$ & ${ }^{56} \mathrm{Co}$ \\
$14.41^{\mathrm{b}}$ & $1.19 \times 10^{-3}$ & $7.80 \times 10^{-7}$ & ${ }^{57} \mathrm{Co}$ \\
122.06 & $1.03 \times 10^{-2}$ & $6.79 \times 10^{-6}$ & ${ }^{57} \mathrm{Co}$ \\
136.47 & $1.19 \times 10^{-3}$ & $7.80 \times 10^{-7}$ & ${ }^{57} \mathrm{Co}$ \\
\hline
\end{tabular}

Notes.

${ }^{a}$ Flux is in units of photon $\mathrm{s}^{-1} \mathrm{~cm}^{-2}$

${ }^{b}$ Line is outside the energy range used for fitting.

A more quantitative statement on the amount of ${ }^{56} \mathrm{Ni}$ synthesized during explosion and on the properties of the ejecta can be obtained from the comparison of the data with the predictions of the models. Since the late period is less affected by the transparency of the ejecta we start our analysis with the total spectrum obtained by INTEGRAL over this period.

\subsubsection{Late Data}

The results of fitting of the combined ISGRI+SPI spectrum (Figure 8) for the late period are given in Table 4. A full set of models from Table 2 is used. The two groups of columns in Table 4 differ by the energy range in the SPI data used for comparison with the model. In the first group the data of ISGRI (70-600 keV) and SPI (400-1350 keV) are used. The data below $70 \mathrm{keV}$ are likely contaminated by other sources in M82. The SPI data below $400 \mathrm{keV}$ are omitted since during the late period the data at these energies are expected to be dominated by the off-diagonal response of SPI. That is, the observed SPI spectrum below $500 \mathrm{keV}$ includes a significant contribution from the gamma-ray photons at higher energies, which are down-scattered inside the body of the telescope (see Figure 2). The Null model (no source) gives $\chi^{2}=1945.38$ for 1906 spectral bins. The improvement of the $\chi^{2}$ relative to the Null is calculated by fixing the normalization at the predicted value for $D=3.5 \mathrm{Mpc}$ (column 2) and by letting it vary (columns 3 and 4). The typical value of $\Delta \chi^{2} \sim 65$ suggests $\sim 8 \sigma$ detection.

One can draw two conclusions from this exercise. First of all a set of canonical 1D deflagration (W7) or delayed-detonation models (e.g., DD4) fit the data well without any adjustments to the normalization. The pure detonation model DETO and a subChandrasekhar model HED 6 give a poor fit and overproduce or underproduce the observed flux, respectively. Second, once the normalization is allowed to vary, all models give almost identical gain in $\chi^{2}$, suggesting that the relative strength of all prominent features is comparable in all models. Given the uncertainty in the distance to SN 2014J (or M82) a deviation of the normalization at the level of $\sim 20 \%$ cannot be excluded. But
DETO and HED6 models require far larger changes in the normalization.

While the SPI data with $E<400 \mathrm{keV}$ have been omitted in the above analysis to concentrate on the data less affected by the off-diagonal response, the right part of Table 4 extends the analysis down to $70 \mathrm{keV}$ for both instruments. The basic conclusions remain the same, although, as expected, the significance of the detection increases to $\gtrsim 9 \sigma$.

\subsubsection{Early Data}

We now proceed with the same analysis of the early data. The combined ISGRI + SPI spectrum for this period is shown in Figure 9. Table 5 contains the gain in $\chi^{2}$ for the same set of models.

The DETO is clearly inconsistent with the data-inclusion of the model increases $\chi^{2}$ relative to the Null model (no source). The HED 6 model, which gave a poor fit to the late data, yields a $\chi^{2}$ comparable to other models. This is because the smaller amount of ${ }^{56} \mathrm{Ni}$ is compensated by larger transparency of the lower-mass ejecta, which is important for the early data.

The 3Dbbal model gives poor gain in $\chi^{2}$ if the normalization is fixed and the SPI data below $400 \mathrm{keV}$ are excluded. If the normalization is free, and, especially, if the SPI data below $400 \mathrm{keV}$ are included, this model performs marginally better than other models. However, it performs significantly better than other models when the SPI data below $400 \mathrm{keV}$ are included. This is not surprising, since the $3 \mathrm{Dbbal}$ model has been designed to fit the SPI data during this period (see Isern et al. 2015 for details). The different "ranking" of the 3Dbbal model seen in Table 5 when SPI data below $400 \mathrm{keV}$ are included or excluded suggests a tension in the comparison of the fixed-normalization 3Dbbal model with the SPI and ISGRI data and also with the SPI data below and above $400 \mathrm{keV}$ (see below).

\subsubsection{Early and Late Data Together}

Finally, in Table 6, we compare jointly the early and late data of ISGRI and SPI with the models calculated for corresponding periods. The two columns in Table 6 differ by the energy range in the SPI data used for comparison with the model. In each case the normalization was fixed at the value set by the adopted distance of $3.5 \mathrm{Mpc}$. In each column we mark with bold face the models that have $\Delta \chi^{2}$ different from the model with the largest $\Delta \chi^{2}$ by less than 4 (see the Appendix for clarification on the interpretation of this criterion in Bayesian and frequentist approaches). Once again, the 1D deflagration model $\mathrm{W} 7$ and "standard" delayed-detonation model perform well. The $3 \mathrm{Dbbal}$, which was designed to account for a tentative feature in the early SPI data at low energies, not surprisingly performs well if the SPI data below $400 \mathrm{keV}$ are included. However, if only the data above $400 \mathrm{keV}$ are used for SPI, this model yields significantly lower $\Delta \chi^{2}$ than the $\mathrm{W} 7$ or DDT1p1 models.

\subsection{Comparison of Gamma-ray Light Curves with Models}

While the spectra for the early and late periods already provide an overall test of the basic models, additional information can be obtained by analyzing the time variations of the fluxes in broad energy bands (see Figures 3 and 4). The total number of time bins is 34 . Each bin corresponds to one revolution (i.e., $\sim 3$ days). The first row in Table 7 provides the values of $\chi^{2}$ (for the Null model of no source) in three energy 


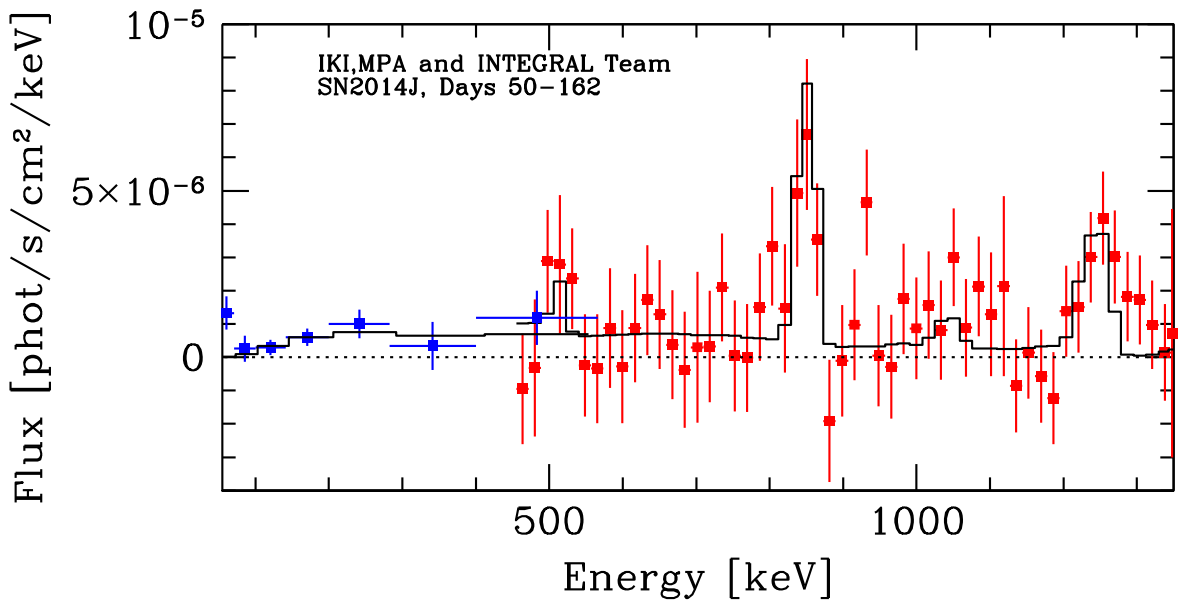

Figure 8. Combined ISGRI/SPI spectrum for the late period. The model (W7, see Table 2) has been convolved with the SPI off-diagonal response. The SPI data below $450 \mathrm{keV}$ are omitted since during the late period the data at these energies are expected to be dominated by the off-diagonal response of SPI.

Table 4

$\Delta \chi^{2}$ for Basic Models for Fixed and Free Normalization Relative to the Null Model of no Source for the Late Period

\begin{tabular}{|c|c|c|c|c|c|c|}
\hline \multirow{2}{*}{$\begin{array}{l}\text { Data Set: } \\
\text { Model }\end{array}$} & \multicolumn{3}{|c|}{ ISGRI(70-600 kev) and SPI(400-1350 kev) } & \multicolumn{3}{|c|}{ ISGRI(70-600 keV) and SPI(70-1350 keV) } \\
\hline & $\overline{N=1, \Delta \chi^{2}}$ & $N_{\text {free }}$ & $\overline{\Delta \chi^{2}}$ & $\overline{N=1, \Delta \chi^{2}}$ & $N_{\text {free }}$ & $\Delta \chi^{2}$ \\
\hline DDT1p1 & 66.4 & $1.03 \pm 0.13$ & 66.5 & 87.3 & $1.09 \pm 0.12$ & 87.9 \\
\hline DDTe & 62.1 & $1.09 \pm 0.14$ & 62.5 & 82.3 & $1.15 \pm 0.13$ & 83.7 \\
\hline DETO & 10.1 & $0.52 \pm 0.06$ & 66.4 & 30.2 & $0.55 \pm 0.06$ & 87.7 \\
\hline HED 6 & 47.8 & $1.86 \pm 0.24$ & 60.7 & 60.1 & $2.01 \pm 0.22$ & 80.5 \\
\hline W7 & 65.0 & $0.94 \pm 0.12$ & 65.3 & 86.9 & $1.01 \pm 0.11$ & 86.9 \\
\hline DD 4 & 64.7 & $0.89 \pm 0.11$ & 65.7 & 87.0 & $0.95 \pm 0.10$ & 87.3 \\
\hline No source, $\chi^{2}$ (d.o.f.) & & $1945.4(1906)$ & & & $2696.9(2566)$ & \\
\hline
\end{tabular}

Note. $N$ is the normalization of the model with $N=1$ corresponding to the explosion at the distance of $3.5 \mathrm{Mpc} . \Delta \chi^{2}$ characterizes an improvement of $\chi^{2}$ for a given model relative to the Null model. Larger positive values indicate that the model is describing the data significantly better than other models (see the Appendix). The data below $70 \mathrm{keV}$ are likely contaminated by other sources in M82. SPI data below $400 \mathrm{keV}$ are omitted in the first data set (left half of the table) since the data at these energies are expected to be dominated by the off-diagonal response of SPI (see Section 3.1).

Table 5

As in Table 4 but for the Early Period

\begin{tabular}{|c|c|c|c|c|c|c|}
\hline \multirow{2}{*}{$\begin{array}{l}\text { Data Set: } \\
\text { Model }\end{array}$} & \multicolumn{3}{|c|}{ ISGRI(70-600 keV) and SPI(400-1350 keV) } & \multicolumn{3}{|c|}{ ISGRI(70-600 keV) and SPI(70-1350 keV) } \\
\hline & $N=1, \Delta \chi^{2}$ & $N_{\text {free }}$ & $\Delta \chi^{2}$ & $N=1, \Delta \chi^{2}$ & $N_{\text {free }}$ & $\Delta \chi^{2}$ \\
\hline DDT1p1 & 14.9 & $0.84 \pm 0.21$ & 15.4 & 33.2 & $1.11 \pm 0.19$ & 33.5 \\
\hline $\mathrm{DDTe}$ & 14.3 & $1.30 \pm 0.33$ & 15.1 & 26.9 & $1.72 \pm 0.30$ & 32.6 \\
\hline DETO & -83.9 & $0.28 \pm 0.07$ & 14.8 & -64.8 & $0.37 \pm 0.06$ & 35.2 \\
\hline HED 6 & 15.7 & $1.05 \pm 0.26$ & 15.8 & 32.7 & $1.39 \pm 0.23$ & 35.5 \\
\hline 3Dbbal & 6.7 & $0.56 \pm 0.13$ & 17.6 & 37.0 & $0.76 \pm 0.12$ & 41.4 \\
\hline DD4 & 14.1 & $0.77 \pm 0.19$ & 15.5 & 33.6 & $1.01 \pm 0.17$ & 33.6 \\
\hline No source, $\chi^{2}$ (d.o.f.) & & 1856.7 (1906) & & & $2615.9(2566)$ & \\
\hline
\end{tabular}

bands: $\quad 100-200 \mathrm{keV}$ (ISGRI), $835-870 \mathrm{keV}$ (SPI), and 1220-1272 keV (SPI). The normalization of the model light curves is fixed to 1 . For 34 bins the value of $\chi^{2}$ for a correct model is expected to be in the interval $\sim 26-42$ in $68 \%$ of cases. Clearly, the Null model does not fit the data well.

Other rows show the improvement in $\chi^{2}$ relative to the Null model, i.e., $\Delta \chi^{2}=\chi_{\text {Null }}^{2}-\chi_{\text {model }}^{2}$. From Table 7 it is clear that the DETO model strongly overpredicts the flux in all bands and can be excluded ( $\chi^{2}$ becomes worse when this model is used). Other models leads to significant improvement with respect to the Null model, except for the $3 \mathrm{Dbb} b \mathrm{l}$ model in the $100-200 \mathrm{keV}$ band where it exceeds the observed flux in the early observation, while in the SPI bands all these models are comparable.

The last column in Table 7 provides $\chi^{2}$ for the three bands jointly. This is basically the sum of the values of $\chi^{2}$ for 
Table 6

$\Delta \chi^{2}$ for the Joint Data Set of the Early and Late Spectra for a Basic Set of Models with Fixed Normalization

\begin{tabular}{lcc}
\hline \hline Model & $\begin{array}{c}\text { ISGRI and SPI } \\
(400-1350 \mathrm{keV})\end{array}$ & $\begin{array}{c}\text { ISGRI and SPI } \\
(70-1350 \mathrm{keV})\end{array}$ \\
\hline DDT1p1 & $\Delta \chi^{2}$ & $\Delta \chi^{2}$ \\
DDT1p4halo & $\mathbf{8 1 . 3}$ & $\mathbf{1 2 0 . 5}$ \\
DDTe & $\mathbf{8 0 . 5}$ & 117.8 \\
DETO & 76.4 & 109.2 \\
HED6 & -73.8 & -34.7 \\
W7 & 63.5 & 92.8 \\
ddt1p4 & $\mathbf{8 0 . 9}$ & $\mathbf{1 2 1 . 7}$ \\
3Dbbal & 76.2 & $\mathbf{1 2 0 . 7}$ \\
DD4 & 69.9 & $\mathbf{1 2 2 . 7}$ \\
\hline
\end{tabular}

Note. Bold-face type is used for models that have $\Delta \chi^{2}$ different from the model with the largest $\Delta \chi^{2}$ by less than 4 , the criterion used to group models into "more plausible" and "less plausible" (see the appendix). The value of $\Delta \chi^{2}$ shows the improvement in $\chi^{2}$ relative to the null model of no source.

individual bands. Bold-face type is used for the bestperforming models: $W 7$ and DDT1P1. As in Section 5.1, these are the models that have $\Delta \chi^{2}$ different from the model with the largest $\Delta \chi^{2}$ by less than 4 (see the Appendix).

One can also compare the light curves with the hypothesis of a constant flux. The mean level of flux was estimated for each band and the value of $\chi^{2}$ was calculated. The values of $\Delta \chi^{2}$ relative to "No source" are given in the last row of Table 7. One can see that this simple model is almost as good as other bestperforming models in individual bands (even taking into account that this model has a free parameter-the mean flux). This is of course the result of low statistical significance of the SN 2014J detection that makes it difficult to constrain time variations of a faint signal. For the combined values for all three bands the effective number of free parameters is three (the mean fluxes in each band), and one can conclude that, for example, the $\mathrm{W} 7$ model performs marginally better than the constant flux model.

\subsection{Search for Velocity Substructure in the Late Data}

The above analysis suggests that the INTEGRAL data broadly agree with a subset of simple 1D models (e.g., W7 or DD4). Since the true structure of SN 2014J is surely more complicated than predicted by $1 \mathrm{D}$ models, it is interesting to verify whether adding an extra component to the model (on top of the best-performing W7 model) significantly improves the fit. In this section, we use the TEM model as such an extra component. This choice is partly driven by the discussion of the possible presence of ${ }^{56} \mathrm{Ni}$ at or near the surface of the ejecta in Diehl et al. (2014) and Isern et al. (2015). As described in Section 4.2, the TEM model described a transparent clump of radioactive Ni. All gamma-ray lines associated with the $\mathrm{Ni} \rightarrow$ $\mathrm{Co} \rightarrow \mathrm{Fe}$ decay in the TEM model are tied to the energy (redshift) and the width of the reference $847 \mathrm{keV}$ line. The flux ratios are also tied together using a model of an optically thin clump, taking into account time evolution of the $\mathrm{Ni}$ and $\mathrm{Co}$ masses. Examples of spectra predicted by the TEM model (for $1 M_{\odot}$ of ${ }^{56} \mathrm{Ni}$ ) are shown in Figure 10 .

Thus, we consider a composite model, comprising the $\mathrm{W} 7$ model (with the normalization fixed to 1) and the TEM model. This two-component (W7+TEM) model effectively searches for a transparent clump of radioactive material on top of the baseline $\mathrm{W} 7$ model (see Figure 11). The horizontal axis shows the energy of the reference $847 \mathrm{keV}$ line in the observer frame and different colors correspond to different $847 \mathrm{keV}$ line broadenings parameterized through a Gaussian $\sigma$-see legend. For a given redshift/energy and width of the reference $847 \mathrm{keV}$ line the model has the normalization (initial ${ }^{56} \mathrm{Ni}$ mass) as the only free parameter. The best-fitting ${ }^{56} \mathrm{Ni}$ mass is shown in the top panel of Figure 11. The bottom panel of Figure 11 shows the improvement in $\chi^{2}$ (relative to the $\mathrm{W} 7$ model alone) due to the TEM model.

As is clear from Figure 11 this model does not provide compelling evidence for a transparent clump on top of the $\mathrm{W} 7$ in the late data. Formally, there is a $\Delta \chi^{2} \sim 9.5$ peak at $\sim 858.5 \mathrm{keV}$, which corresponds to a narrow $(\sim 1 \mathrm{keV}$ broad, red curve) component with a negative mass of $-0.05 M_{\odot}$, which can be interpreted as marginal evidence for a dip in the velocity substructure, given that this improvement in $\Delta \chi^{2}$ came at the cost of adding three more parameters ${ }^{14}$ to the model. One can estimate the constraints on the line flux (mass of a transparent clump) that such analysis can provide, by fixing the centroid energy and the width of the reference $847 \mathrm{keV}$ line and calculating the expected statistical uncertainty. Since the normalization of the TEM model is the only free parameter in this particular experiment, the estimation of the uncertainty is straightforward (see Figure 12). Three curves shown in Figure 12 show $1 \sigma$ uncertainty on the initial ${ }^{56} \mathrm{Ni}$ mass for the early set (dashed blue: SPI data in the 70-1350 keV band; long-dashed green: 400-1350 keV) and late set (solid red: 400-1350 keV). A conservative upper limit based on the assumption of pure statistical errors would be three times these values. Letting the broadening and the redshift be free parameters (the lookelsewhere effect) would increase this limit even further.

These experiments show that the late data are consistent with the presence of a velocity substructure (parameterized via our TEM model) on top of the 1D W7 model at the level $\sim 0.05 M_{\odot}$, provided that the lines are slightly broadenened.

We now do a similar experiment with the early data, using a $\mathrm{TEM}+\mathrm{W} 7$ model for SPI data in the $400-1350 \mathrm{keV}$ band (Figure 13, left panel) and in the 70-1350 keV band (Figure 13, right panel).

The left panel does not show any significant evidence for a clump on top of the $\mathrm{W} 7$ model. The structure in the right panel is more complicated. The data used in this panel now include the ${ }^{56} \mathrm{Ni}$ line at $158 \mathrm{keV}$. We note that if the $158 \mathrm{keV}$ line is able to escape, then the higher energy lines of $\mathrm{Ni}$ and $\mathrm{Co}$ certainly can too. Therefore the analysis should be done for the whole band to achieve the most significant results. First of all, our analysis does not show compelling evidence for a narrow and unshifted component reported in Diehl et al. (2014); there is a weak peak ( $\Delta \chi^{2} \sim 6$, i.e., $\sim 2.4 \sigma$ detection if we ignore the freedom in the redshift and broadening) at $847.5 \mathrm{keV}$, corresponding to a narrow line (black curve) with a mass of $\sim 0.027 M_{\odot}$ of ${ }^{56} \mathrm{Ni}$. There are several separate peaks of similar magnitude, covering the energy range of interest. However, there is more significant (albeit also marginal) evidence for a redshifted and broad component with $M_{\mathrm{Ni}} \sim 0.08 M_{\odot}, E \sim$ $826.5 \mathrm{keV}$ and $\sigma \sim 8 \mathrm{keV}$ (see Isern et al. 2015 for discussion). The gain in $\chi^{2}$ is $\sim 18$, and for a fixed energy and broadening (putting to one side possible systematic errors in the

\footnotetext{
${ }^{14}$ We note that the width and especially energy of the reference line are very nonlinear parameters that could lead to large changes in $\chi^{2}$.
} 
Table 7

$\Delta \chi^{2}$ for Light Curves in Three Energy Bands for Different Models

\begin{tabular}{|c|c|c|c|c|}
\hline Model & 100-200 keV (ISGRI) & $835-870 \mathrm{keV}$ (SPI) & $1220-1272 \mathrm{keV}$ (SPI) & Three Bands Jointly \\
\hline$\overline{\text { No } \text { source, } \chi^{2}}$ & 51.0 & 49.6 & 51.1 & 151.7 \\
\hline DDT1P1 & 16.9 & 18.6 & 19.3 & 54.8 \\
\hline DDT1P4halo & 9.2 & 17.4 & 19.5 & 46.1 \\
\hline DETO & -105.0 & -6.3 & 13.5 & -97.8 \\
\hline HED 6 & 20.6 & 16.0 & 14.1 & 50.7 \\
\hline W7 & 18.8 & 18.4 & 20.0 & 57.2 \\
\hline $\mathrm{DD} 4$ & 12.7 & 17.9 & 20.0 & 50.6 \\
\hline CONST & 18.7 & 16.1 & 18.5 & 53.3 \\
\hline
\end{tabular}

Note. The total number of time bins is 34. For the joint $\chi^{2}$ the effective number of bins is three times larger, i.e.,102. The value of $\Delta \chi^{2}$ shows the improvement in $\chi^{2}$ relative to the Null model of no source. The value of $\chi^{2}$ for the Null model is given in the first row.

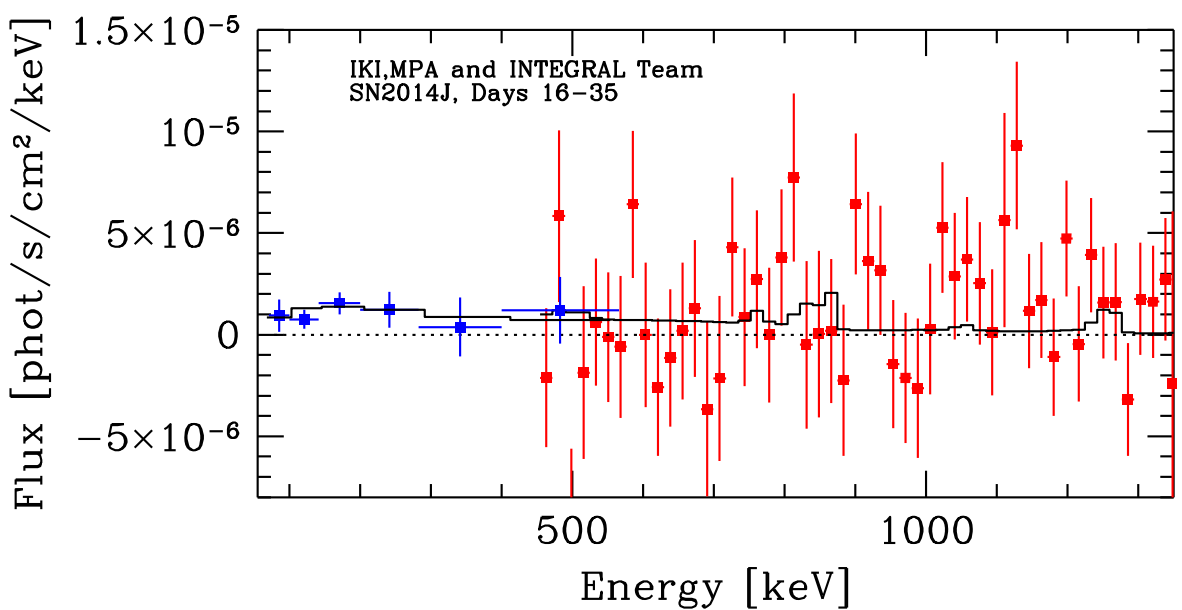

Figure 9. Combined ISGRI/SPI spectrum for the early period. The model (W7, see Table 2) has been convolved with the SPI off-diagonal response.

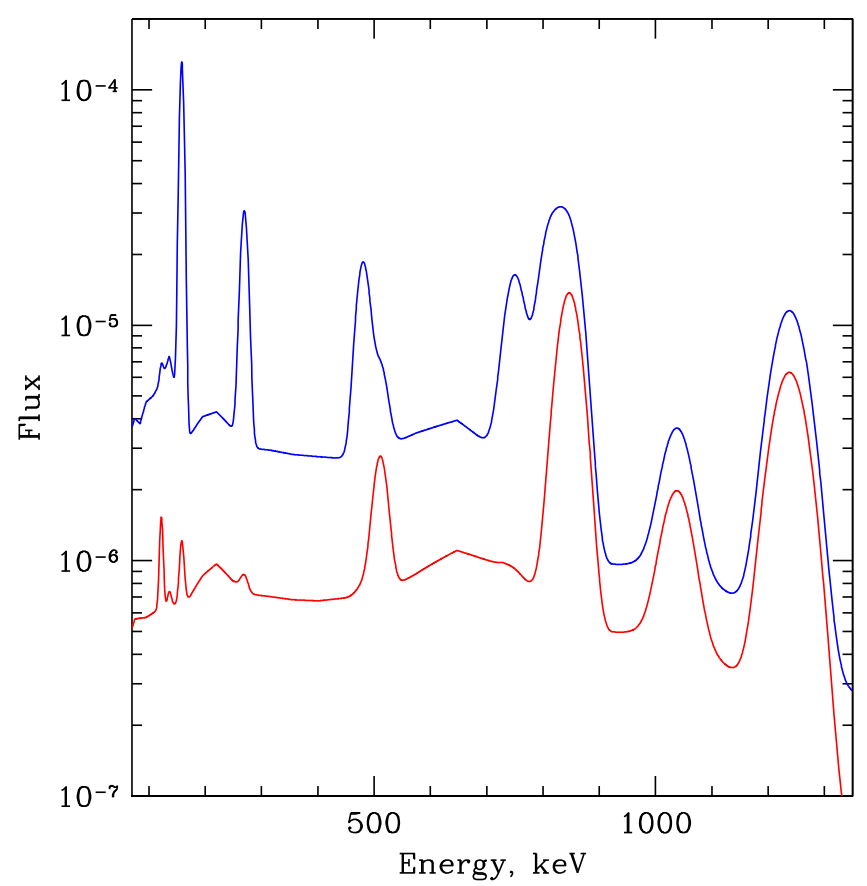

Figure 10. Spectra predicted by the TEM model for early (blue) and late (red) data sets, convolved with the SPI response. The broadening of the reference $847 \mathrm{keV}$ line is set to $20 \mathrm{keV}$ (Gaussian sigma). The initial ${ }^{56} \mathrm{Ni}$ mass is $1 M_{\odot}$. background modeling and uncertainties in the calibration of the off-diagonal response) this would be a $4.2 \sigma$ detection. However, the freedom in the energy, width (look-elsewhere effect), and normalization reduces the significance. Should all these free parameters be linear (as normalization is), one would expect a change in $\chi^{2}$ of $\sim 3$ due to purely statistical fluctuations. However, the energy and the width are nonlinear and the gain in $\chi^{2}$ might be significantly larger. In Figures 11 and 13 we see multiple peaks with the change/gain in $\chi^{2}$ up to $\sim 10$. Assuming that the latter value can be used as a crude estimate of a possible gain in $\chi^{2}$ due to nonlinearity of the TEM model, the significance of the detection of the excess drops below $3 \sigma$.

Taking the best-fitting parameters at face value, we can go back to the late data and compare the spectra (in the 400-1350 keV band) with the TEM+W7 model, freezing TEM model parameters at the best-fitting values obtained for the early data. This gives $\chi^{2}=1883.05$, i.e., worse than the $\mathrm{W} 7$ model alone $\left(\chi^{2}=1879.3\right)$. If we let the normalizations of both TEM and $W 7$ models vary (but freeze energy and broadening of the TEM model), then we improve $\chi^{2}$ slightly to 1878.9 , but the best-fitting mass becomes slightly negative, although consistent with zero, $-3 \times 10^{-3} \pm 5 \times 10^{-2} M_{\odot}$, while the best-fitting normalization of the $\mathrm{W} 7$ model becomes 0.92 (see Table 4 where SPI data are used together with the ISGRI data). 


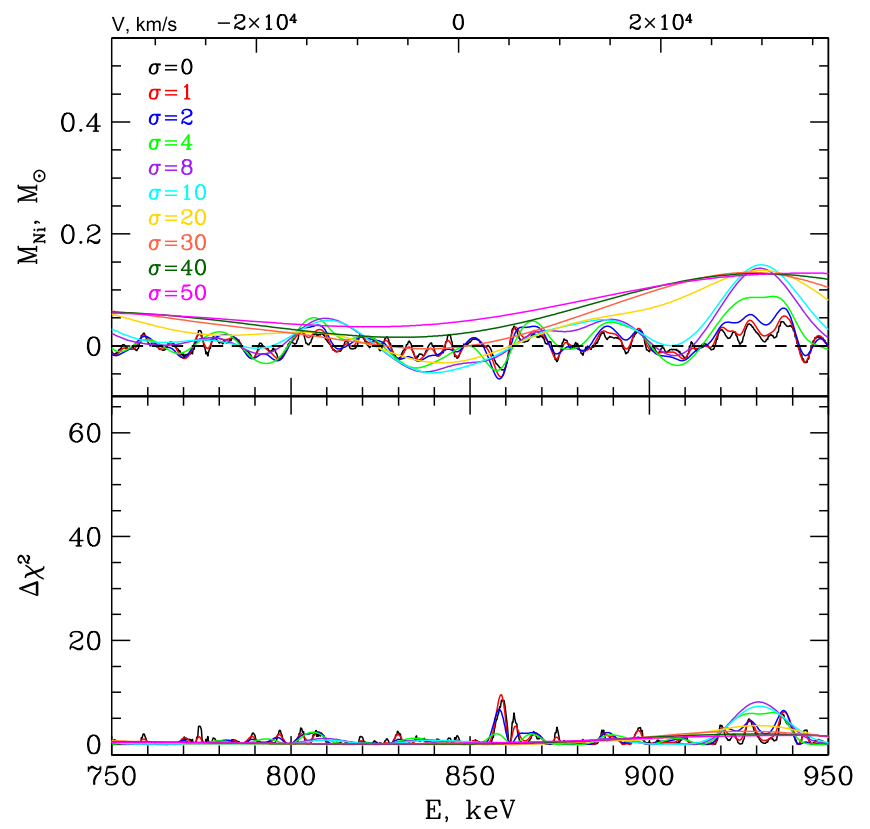

Figure 11. Fitting the SPI data in the 400-1350 keV band with a composite $W 7$ +TEM model. The normalization of the W7 model is fixed to 1 . In the TEM model all lines are tied to the energy (redshift) and the width of the reference $847 \mathrm{keV}$ line. The flux ratios are tied using a model of an optically thin clump, taking into account time evolution of the $\mathrm{Ni}$ and $\mathrm{Co}$ masses. This setup is optimized for a search for a transparent clump on top of the $\mathrm{W} 7$ model). For a given energy and width the model has only normalization (initial ${ }^{56} \mathrm{Ni}$ mass in the clump) as a free parameter. The bottom panel shows the improvement in $\chi^{2}$ and the top panel shows the best-fitting ${ }^{56} \mathrm{Ni}$ clump mass. Different colors correspond to a different $847 \mathrm{keV}$ line broadenings parameterized through a Gaussian $\sigma$-see legend. No compelling evidence for a clump is seen in the data. The sensitivity of the data to the mass of the clump strongly depends on the broadening of the lines (see Figure 12).

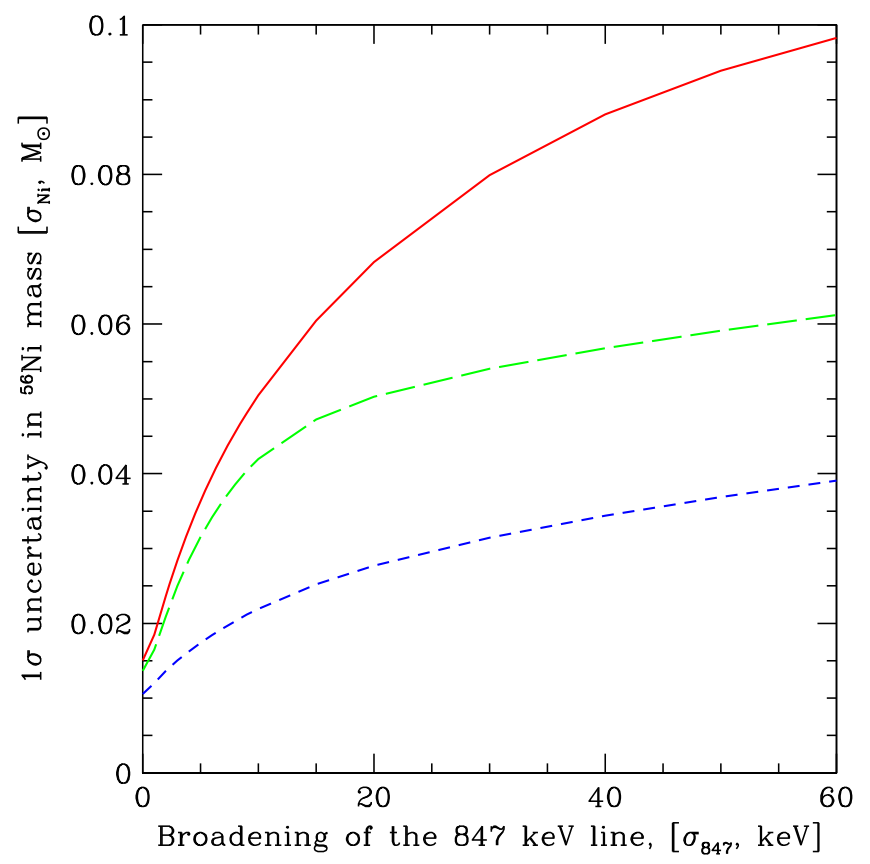

Figure 12. Uncertainty in the initial ${ }^{56} \mathrm{Ni}$ mass as a function of line broadening for the early set (dashed blue: SPI data in the 70-1350 keV band; long-dashed green: $400-1350 \mathrm{keV}$ ) and late set (solid red: 400-1350 keV), assuming transparency to gamma rays generated close to the surface. A conservative upper limit on the initial mass of "extra" radioactive ${ }^{56} \mathrm{Ni}$ is three times this value at a given line width. For the line broadening of $10^{4} \mathrm{~km} \mathrm{~s}^{-1}$ (FWHM), the expected value of $\sigma_{847}$ is $\sim 12 \mathrm{keV}$. This value can be regarded as a fiducial value for a simple SN Ia model.
We conclude that there is a tension between "low" energy SPI data in early observations and the rest of the INTEGRAL data (Tables 5 and 7). However, this tension is not prohibitively large and could be attributed to statistical fluctuations in the data if a conservative approach is adopted. Possible evidence for the redshifted and broadened $158 \mathrm{keV}$ line in the early data and possible implications are further discussed in Isern et al. (2015).

\subsection{PAR Model}

Apart from the models discussed above, we also used the 3PAR model introduced in Churazov et al. (2014b). This is a spherically symmetric model of homologously expanding ejecta with exponential density profile $\rho \propto e^{v / V_{\mathrm{e}}}$. The model is characterized by three parameters: initial mass of the ${ }^{56} \mathrm{Ni} M_{\mathrm{Ni}}$, total mass of the ejecta $M_{\text {ejecta }}$, and characteristic expansion velocity $V_{\mathrm{e}}$ in the exponential density distribution. In this model the mass-weighted root-mean-square velocity of the ejecta is $\sqrt{12} V_{\mathrm{e}}$.

The main shortcoming of this model is the assumption that all elements, including radioactive $\mathrm{Ni}$ and $\mathrm{Co}$, are uniformly mixed through the entire ejecta. This is an ad-hoc assumption, made in order to retain a model with only three parameters, but it is not justified. It has a major impact on the early gamma-ray light curve, producing gamma-ray emission even in the very early phase (see Figures 1, 3, 4). At later times (day 50 or later), the role of mixing is less significant. We therefore applied this model to the late ISGRI and SPI spectra to get estimates of $M_{\mathrm{Ni}}, M_{\text {ejecta }}$, and $V_{\mathrm{e}}$, which are not limited to values characteristic of the set of plausible models given in Table 2. The main purpose of using this model is to understand the level of constraints provided by the INTEGRAL data on the main characteristics of the SN. Simplicity of the model allows us to calculate it on a large grid of possible values of $M_{\mathrm{Ni}}, M_{\text {ejecta }}$, and $V_{\mathrm{e}}$.

A Monte-Carlo radiative transfer code is used to calculate the emergent spectrum, which includes full treatment of Compton scattering (coherent and incoherent) and photoabsorption. Pair production by $\gamma$-ray photons is neglected. The positrons produced by $\beta^{+}$decay annihilate in place via positronium formation. Both two-photon annihilation into the $511 \mathrm{keV}$ line and the ortho-positronium continuum are included.

The results are shown in Figure 14. The best-fitting values $M_{\mathrm{Ni}}=0.63 M_{\odot}, M_{\text {ejecta }}=1.8 M_{\odot}, V_{\mathrm{e}}=3 \times 10^{3} \mathrm{~km} \mathrm{~s}^{-1}$ are marked with a cross. The $1 \sigma$ confidence contours (corresponding to $\Delta \chi^{2}=1$, i.e., for a single parameter of interest) are shown with the thick solid line. Clearly, the Ni mass $M_{\mathrm{Ni}}$ and the characteristic expansion velocity $V_{\mathrm{e}}$ are better constrained than the ejecta mass. This is not surprising given that the data averaged over the period 50-162 days after explosion are used, when the ejecta are relatively transparent to gamma rays. As a result the flux in the lines depends primarily on the $\mathrm{Ni}$ mass, line broadening is set by the expansion velocity, while ejecta mass mostly influences the amplitude of the scattered component, which declines with time relative to the orthopositronium continuum when the optical depth decreases. If we fix the poorly constrained ejecta mass to $M_{\text {ejecta }}=1.4 M_{\odot}$, then the derived $\mathrm{Ni}$ mass is constrained to the range $0.54-0.67 M_{\odot}$.

For the set of models listed in Table 2 we can estimate the effective $V_{\mathrm{e}}$ using the relation $V_{\mathrm{e}}=\sqrt{\frac{E_{K}}{6 M_{\text {ejecta }}}}$, valid for a pure 

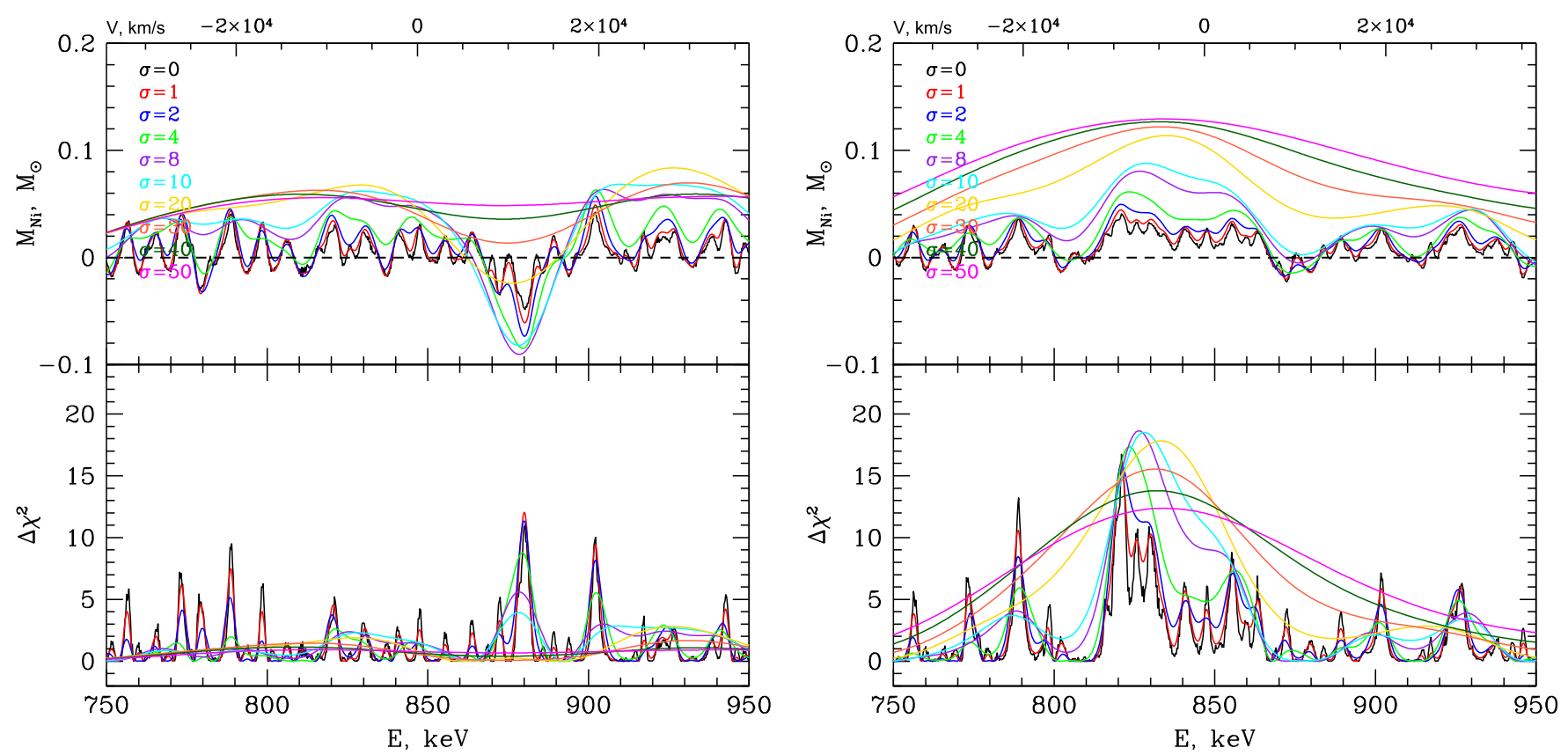

Figure 13. Same as in Figure 11, but for the early SPI spectrum. Left: TEM+W7 model and the SPI data in the 400-1350 keV band. The normalization of the W7 model is fixed to 1. Right: TEM+W7 model and the SPI data in the 70-1350 keV band. The normalization of the $W 7$ model is fixed to 1 . The low-energy part of the SPI spectrum is included to make sure that the ${ }^{56} \mathrm{Ni}$ line at $158 \mathrm{keV}$ is within the energy range probed. There is marginal evidence of a redshifted (by $\sim 8000 \mathrm{~km} \mathrm{~s} \mathrm{~s}^{-1}$ ) component with a width of $\sim 8 \mathrm{keV}$ (Gaussian sigma), corresponding to $M_{\mathrm{Ni}} \sim 0.08 M_{\odot}$. See text for the discussion.

exponential model. The value $V_{\mathrm{e}}$ varies from $\sim 2580 \mathrm{~km} \mathrm{~s}^{-1}$ for DDTe to $\sim 2960 \mathrm{~km} \mathrm{~s}^{-1}$ for the DETO model and is equal to $2740 \mathrm{~km} \mathrm{~s}^{-1}$ and $2820 \mathrm{~km} \mathrm{~s}^{-1}$ for W7 and DDT1p1, respectively. Not surprisingly, all "successful" models (e.g., W7 and DDT1P1) have their characteristic parameters well inside the contours plotted in Figure 14, while DETO and HED6 are far outside the contours, primarily because of the Ni mass.

\subsection{Summary of Model Fitting}

The comparison of the INTEGRAL data with the subset of models (see the sections above) allows one to crudely rank the models according to their success in different tests. For each test (data set) we can choose the "best" model, which provides the largest improvement in $\Delta \chi^{2}$ compared to the Null model (or has the smallest $\chi^{2}$ for the light curves). We can then adopt an ad-hoc definition whereby other models that have $\chi^{2}$ different from the best model by 4 (i.e., $\sim 2 \sigma$ confidence) are classified as "good." A similar approach can be applied to the light curves in each band (Table 7), by adding four to the minimal value of $\chi^{2}$ among models. Applying this test to Tables 4-7, we conclude that $\mathrm{W} 7$ and DDT1p1 pass all these tests, closely followed by $\mathrm{DD} 4$, ddt $1 \mathrm{p} 4$, and then by DDT1P4halo and 3Dbbal. DETO and HED6 fail most of the tests. Of course, given the uncertainties in the distance, background modeling, and calibration issues, we cannot reject models other than DETO and HED6. For example, if we let the normalization be a free parameter (equivalent to stating that the distance is highly uncertain) then most of the models become barely distinguishable. We state rather that a whole class of near-Chandrasekhar models provides a reasonable description of the data, with the W7 and DDT1p1 being the most successful, closely followed by a broader group of delayeddetonation models.

\section{CONSISTENCY WITH OPTICAL DATA}

We now make several basic consistency checks of gammaray and optical data, using optical observations taken quasisimultaneously with INTEGRAL observations.

\subsection{Optical and Gamma-ray Luminosities}

We use BVRIJHK photometry reported by Foley et al. (2014) to estimate the bolometric (UVOIR) luminosity of SN 2014J on days 73 and 96 after the explosion. Since the data do not contain the $U$-band photometry, we include the $U$ magnitude recovered on the basis of the $U-B$ color of the dereddened normal SN Ia, SN 2003hv (Leloudas et al. 2009). The SN 2014J fluxes were corrected for extinction using slightly different extinction laws reported by Amanullah et al. (2014) and Foley et al. (2014). The average of the two fluxes for each epoch was then used to produce the integrated flux. To this end we approximated the spectral energy distribution (SED) by the combination of two functions each of which is a smooth broken power law. The SED integration in the range $0.1<\lambda<10 \mu \mathrm{m}$ with a distance of $3.5 \mathrm{Mpc}$ results in the luminosity estimates of $(11 \pm 1) \times 10^{41} \mathrm{erg} \mathrm{s}^{-1}$ on day 73 and $(6.5 \pm 0.6) \times$ $10^{41} \mathrm{erg} \mathrm{s}^{-1}$ on day 96. These values agree well with the estimated amount of deposited energy in the best-fitting 3PAR model: $\sim 1.0 \times 10^{42} \mathrm{erg} \mathrm{s}^{-1}$ and $\sim 5.3 \times 10^{41} \mathrm{erg} \mathrm{s}^{-1}$ for days 73 and 96, respectively. According to this model the fraction of thermalized energy is $\sim 34 \%$ and $\sim 20 \%$ for these dates, respectively.

\subsection{Asymmetry in Late Optical Spectra?}

The issue of asymmetry of SN 2014J ejecta is of vital importance because a strong deviation of the ${ }^{56} \mathrm{Ni}$ distribution from spherical symmetry would affect the interpretation of the gamma-ray data. Generally, asymmetry of the ${ }^{56} \mathrm{Ni}$ distribution is expected in the binary WD merger scenario (Pakmor 

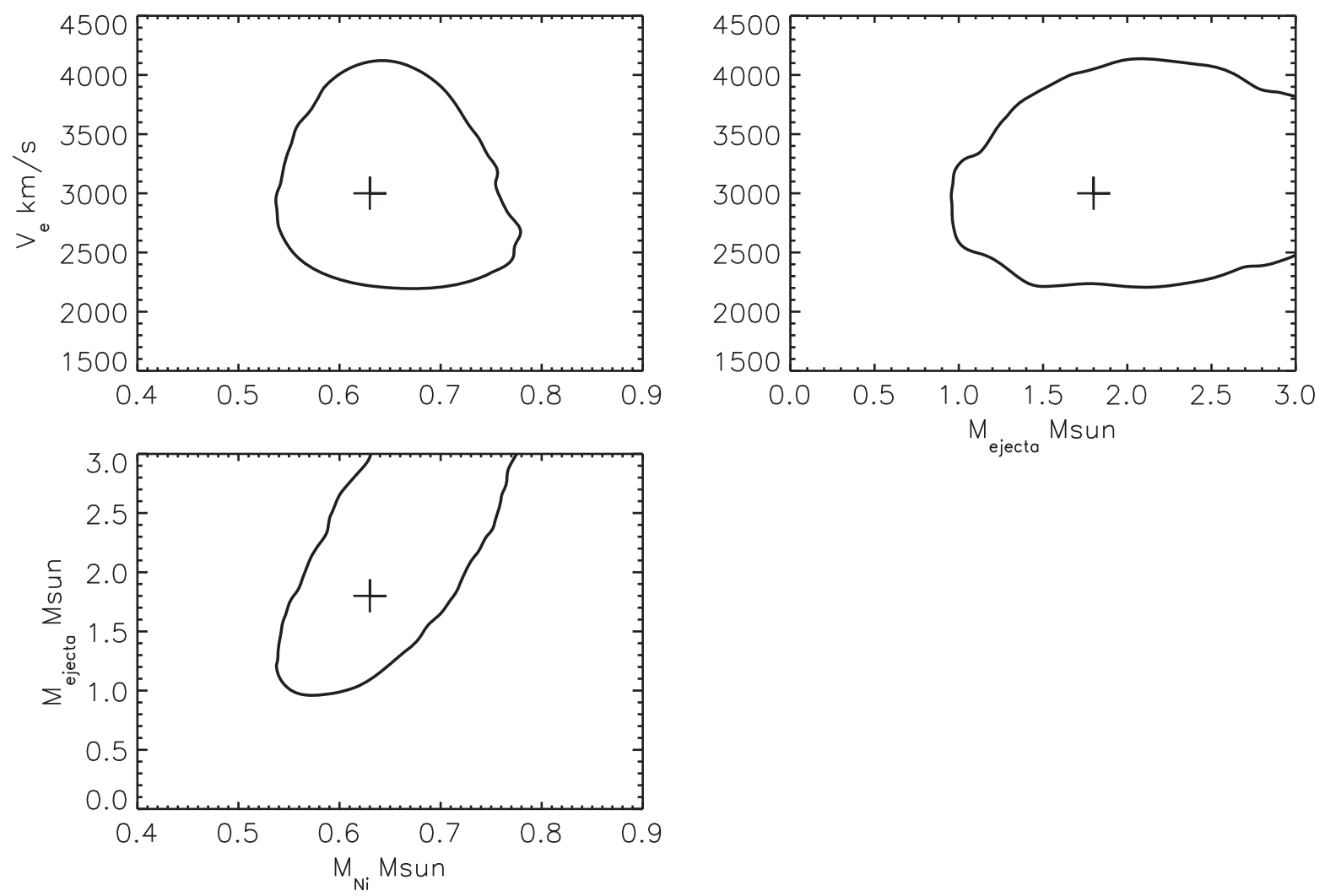

Figure 14. Confidence contours for the 3PAR model, corresponding to $\Delta \chi^{2}=1$ with respect to the best-fitting value. The cross shows the best-fitting parameters of the 3PAR model: $M_{\mathrm{Ni}} \sim 0.63 M_{\odot}, v_{\mathrm{e}} \sim 3000 \mathrm{~km} \mathrm{~s}^{-1}, M_{\text {ejecta }} \sim 1.8 M_{\odot}$. The late ISGRI and SPI spectra are used for this analysis. Confidence intervals plotted in this figure correspond to $1 \sigma$ for a single parameter of interest. The largest uncertainty is in the mass of the ejecta, while the Ni mass is the best determined quantity.

et al. 2012). Moreover, a single-degenerate scenario also does not rule out asymmetry in the ejecta caused by the noncentral early deflagration (Malone et al. 2014). In fact, signatures of asymmetry have already been detected in several SNe Ia at the nebular stage ( $t>100$ days). The asymmetry is manifested in a shift of the emission line and/or in the double-peak emission line profiles (Motohara et al. 2006; Maeda et al. 2010; Dong et al. 2014).

To probe a possible asymmetry of SN 2014J ejecta, we rely on the nebular optical spectrum taken on day 119 after the $B$ maximum, i.e., 136 days after the explosion (Bikmaev et al. 2015) at the $1.5 \mathrm{~m}$ Russian-Turkish telescope (RTT-150) of the TUBITAK National Observatory (Antalya, Turkey). The spectrum of SN 2014J corrected for interstellar reddening in M82 of $E(B-V)=1$ (see Foley et al. 2014) is shown in Figure 15 together with that of SN $2011 \mathrm{fe}$ obtained at the same instrument on day 141 after the maximum. The spectra of the two SNe look similar except for the blueshift of SN $2011 \mathrm{fe}$ emissions by $\sim 10^{3} \mathrm{~km} \mathrm{~s}^{-1}$ relative to SN $2014 \mathrm{~J}$.

We focus on the [Co III] $5890 \AA$ emission that is not hampered markedly by blending with other lines. It should be emphasised that 136 days after the explosion this line is dominated by ${ }^{56} \mathrm{Co}$; the contribution of ${ }^{57} \mathrm{Co}$ and stable $\mathrm{Co}$ isotopes is negligible. The Thomson optical depth at this epoch is small ( $\sim 0.2)$ and does not affect the line profile. The [Co III] emission is the superposition of five lines of the $a^{4} \mathrm{~F}-\mathrm{a}^{2} \mathrm{G}$ multiplet. Each line we describe by a Gaussian with an amplitude proportional to the product of the collisional excitation rate and the radiative branching ratio. We adopt

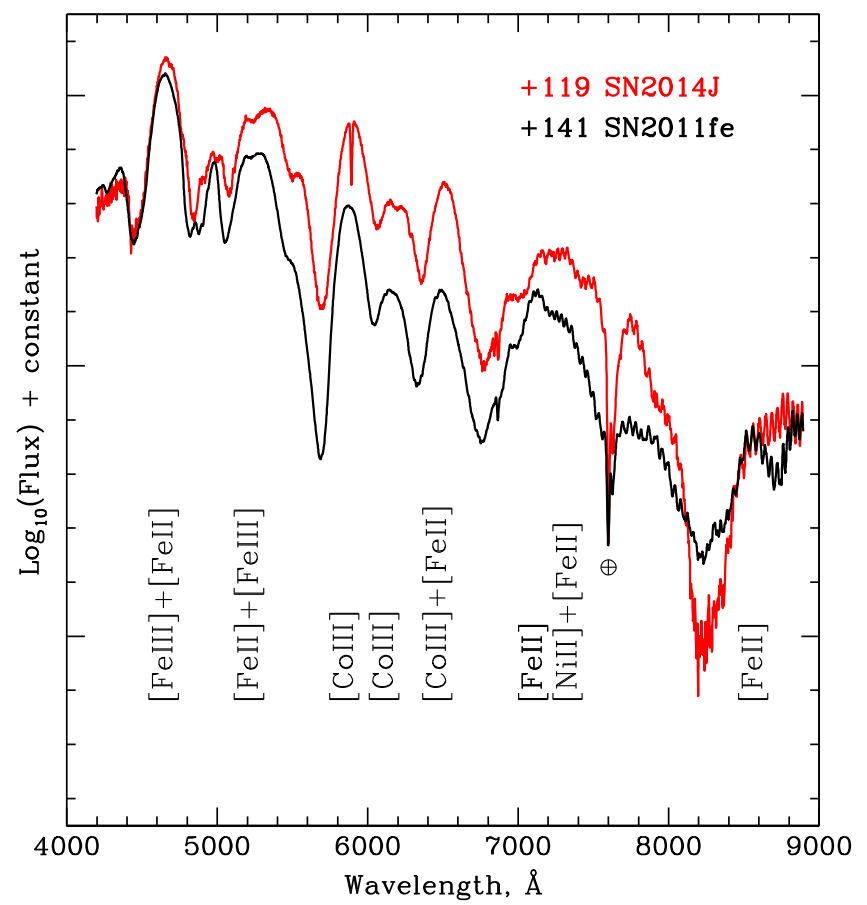

Figure 15. Spectra of SN 2014J (day 119 after the maximum) and SN $2011 \mathrm{fe}$ (day 141 after the maximum) obtained with the RTT-150 telescope (Bikmaev et al. 2015). Overall the spectra are very similar in terms of the flux level, line shape, and line ratios. The exception is the prominent blueshift of [Fe III], [Fe II], and [Co III] emissions of SN 2011fe relative to SN 2014J. The strong interstellar Na I absorption in the SN 2014J spectrum arises in the M82 galaxy. 


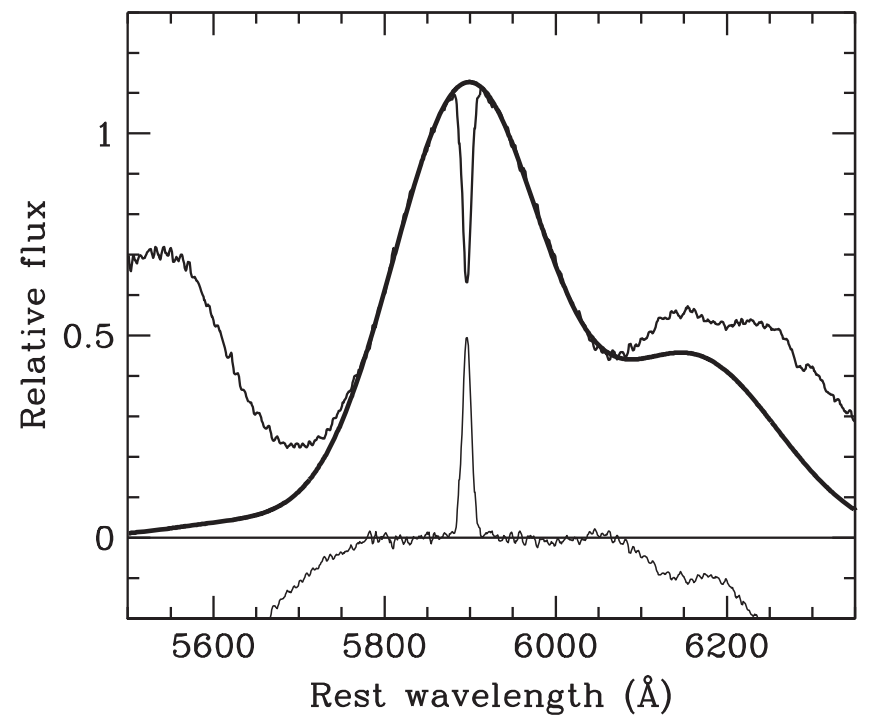

Figure 16. [Co III] $5900 \AA$ A emission in the SN 2014J spectrum on day 119 (thin line) along with the model (thick line) that includes five components of the $\mathrm{a}^{4} \mathrm{~F}-\mathrm{a}^{2} \mathrm{G}$ multiplet. The narrow absorption feature at the top of the profile is due to $\mathrm{Na}$ I interstellar absorption in M82. At the bottom is shown the residual "model minus observation," which demonstrates a good fit in the range 5770-6060 Å.

the heliocentric recession velocity of $+104 \pm 15 \mathrm{~km} \mathrm{~s}^{-1}$ that takes into account the recession velocity of $+203 \mathrm{~km} \mathrm{~s}^{-1}$ for M82 (NASA Extragalactic Database NED) and the rotational velocity of M82 at the position of SN 2014J. The best fit (Figure 16) is found for the full width at half maximum for each line $\mathrm{FWHM}=10450 \mathrm{~km} \mathrm{~s}^{-1}$ and the line shift of $v_{\mathrm{s}}=+130 \pm 17 \mathrm{~km} \mathrm{~s}^{-1}$. With the exception of this small shift, each [Co III] line is fairly symmetric at least in the radial velocity range of $\left|v_{\mathrm{r}}\right|<6100 \mathrm{~km} \mathrm{~s}^{-1}$. The small line shift may be related to either intrinsically small asymmetry of ${ }^{56} \mathrm{Ni}$ distribution or the special viewing angle if the ejecta is actually non-spherical. To summarize, the optical spectrum of SN 2014J does not show signatures of strong asymmetry.

\section{DISCUSSION AND CONCLUSIONS}

We have analyzed a complete set of INTEGRAL observations of SN 2014J. We confirm our previous results (Churazov et al. 2014b) that the data are broadly consistent with the predictions of a nearly Chandrasekhar WD explosion, with (1D) deflagration or delayed-detonation models providing an equally good description (see Tables 6-7). While pure deflagration models are disfavored because of the expected large-scale mixing and incomplete burning in 3D simulations, in the 1D case they yield the same gamma-ray flux as the delayed-detonation models. Pure detonation (or strongly sub-Chandrasekhar) models strongly overproduce (or underproduce) the observed gamma-ray flux and can be excluded. Allowing a freedom in the normalization of the model (equivalent to allowing the initial mass of ${ }^{56} \mathrm{Ni}$ to be a free parameter while keeping other parameters unchanged) makes all models essentially indistinguishable at the level of statistics accumulated by INTEGRAL.

We have searched for possible velocity substructure on top of the predictions from $1 \mathrm{D}$ models, by adding a set of broadened Gaussian lines to the best-performing $\mathrm{W} 7$ model. The energies and fluxes of the lines are tied to the predictions of the $\mathrm{Ni}$ and Co decay chains appropriate for the optically thin clump of Ni. This analysis did not reveal strong evidence for a prominent velocity substructure in the gamma-ray data during the late phase of the SN evolution (after day 50). Given the statistics accumulated by INTEGRAL, a clump with a ${ }^{56} \mathrm{Ni}$ mass $\sim 0.05 M_{\odot}$ producing slightly broadened lines (Figure 12) could be consistent with the late gamma-ray data. Similar analysis of the early data has a best-fitting solution with a redshifted and broadened component with $M_{\mathrm{Ni}} \sim 0.08 M_{\odot}, E \sim$ $826.5 \mathrm{keV}$, and $\sigma \sim 8 \mathrm{keV}$. However, the statistical significance of this extra component is marginal and the late observations do not provide further evidence for the presence of such a component (see also Diehl et al. 2014; Isern et al. 2015, for independent analysis of early observations of SN 2014J).

From the optical light curves and spectra SN 2014J appears to be a "normal" SN Ia with layered structure and no evidence for large-scale mixing (e.g., Ashall et al. 2014; Marion et al. 2015), consistent with the delayed-detonation models. The detection of stable Ni (Friesen et al. 2014; Telesco et al. 2015) in IR suggests a high density of the burning material, characteristic of a near-Chandrasekhar WD.

The optical spectrum taken at the nebular stage (day $\sim 136$ after the explosion) also does not show strong asymmetry in the $\mathrm{Co}$ and $\mathrm{Fe}$ lines. Unless the viewing angle is special, the distribution of these elements in the ejecta is symmetric. These data do not provide any direct support for the collision/merger scenario. The late spectrum of SN 2014J is very similar to that of SN $2011 \mathrm{fe}$, albeit with the pronounced blueshift of emission lines of the latter.

Apart from the above-mentioned feature in the early observation, which we consider as marginal, the rest of the INTEGRAL and optical data appear consistent with the predictions of "canonical" 1D explosion models of a nearly Chandrasekhar carbon-oxygen white dwarf.

This work was based on observations with INTEGRAL, an ESA project with instruments and a science data center funded by ESA member states (especially the principal investigator countries: Denmark, France, Germany, Italy, Switzerland, and Spain) and with the participation of Russia and the United States. We are grateful to ISOC for their scheduling efforts, and the INTEGRAL Users Group for their support in the observations. E.C., R.S., and S.G. are partly supported by grant No. 14-22-00271 from the Russian Scientific Foundation. J.I. is supported by MINECO-FEDER (ESP2013-47637-P) and Generalitat de Catalunya grants. I.B. is partly supported by the Russian Government Program of Competitive Growth of KFU. E.B. is supported by Spanish MINECO grant AYA2013-40545. The SPI project has been completed under the responsibility and leadership of CNES, France. ISGRI has been realized by CEA with the support of CNES. We thank Adam Burrows, Peter Höflich, Rishi Khatri, Ken Nomoto, Victor Utrobin, Alexey Vikhlinin, and Stan Woosley for helpful discussions.

\section{APPENDIX \\ COMPARISON OF SIMPLE MODELS VIA THE $\Delta \chi^{2}$ CRITERION}

In this appendix we clarify our approach of comparing different models using ungrouped spectral and/or timing data.

\section{A.1. Grouping the Data and Small Number of Counts Per Bin}

Consider $N$ data points $D_{i}$ for $i=1, N$, and $N \gg 1$ (e.g., a spectrum measured in $N$ energy bins) that correspond to a 
model $M_{t}, D_{i}=M_{t, i}+n_{i}$, where $n_{i}$ is the noise with Gaussian distribution with zero mean and known variance $\sigma_{i}^{2}$. The noise is uncorrelated, i.e., $\left\langle n_{i} n_{j}\right\rangle=0$ if $i \neq j$. For simplicity, in the following we drop the index $i$ in the expressions containing summation over $i$.

Suppose that we want to compare two competing models $M_{1}$ and $M_{2}$ with no free parameters (this corresponds to a NeymanPearson lemma of two simple hypotheses). For our purpose it is useful to write explicitly the probability distribution of $\Delta \chi^{2}$ between competing models.

Let us define $\delta_{1}=M_{1}-M_{t}$ and $\delta_{2}=M_{2}-M_{t}$ and calculate $\chi^{2}$ for both models. For the model $M_{1}$

$$
\begin{aligned}
\chi_{1}^{2} & =\sum\left(\frac{M_{1}-D}{\sigma}\right)^{2}=\sum\left(\frac{\delta_{1}+n}{\sigma}\right)^{2} \\
& =\sum\left(\frac{\delta_{1}}{\sigma}\right)^{2}+2 \sum \frac{\delta_{1} n}{\sigma^{2}}+\sum\left(\frac{n}{\sigma}\right)^{2},
\end{aligned}
$$

and a similar expression holds for $M_{2}$. Thus $\Delta \chi^{2}=\chi_{1}^{2}-\chi_{2}^{2}$ is

$$
\begin{gathered}
\Delta \chi^{2}=\sum\left(\frac{\delta_{1}}{\sigma}\right)^{2}-\sum\left(\frac{\delta_{2}}{\sigma}\right)^{2} \\
+2 \sum \frac{\delta_{1} n}{\sigma^{2}}-2 \sum \frac{\delta_{2} n}{\sigma^{2}} \\
=\sum\left(\frac{\delta_{1}}{\sigma}\right)^{2}-\sum\left(\frac{\delta_{2}}{\sigma}\right)^{2}+2 \sum \frac{\delta_{1,2} n}{\sigma^{2}},
\end{gathered}
$$

where $\delta_{1,2}=M_{1}-M_{2}$. The last term in the above expression obviously has a Gaussian distribution with zero mean and variance $4 \sum \frac{\delta_{1,2}^{2}<n^{2}>}{\sigma^{4}}=4 \sum \frac{\delta_{1,2}^{2}}{\sigma^{2}}$. Thus

$$
\begin{aligned}
\Delta \chi^{2}= & \sum\left(\frac{\delta_{1}}{\sigma}\right)^{2}-\sum\left(\frac{\delta_{2}}{\sigma}\right)^{2} \\
& +X \sqrt{4 \sum \frac{\delta_{1,2}^{2}}{\sigma^{2}}}
\end{aligned}
$$

where $X$ has a normal distribution. It is clear that the above expression does not depend on data grouping (see Churazov et al. 1996) as long as the grouping does not severely affect the shape of $\delta_{1}, \delta_{2}$, or $\delta_{1,2}$. Furthermore, $\Delta \chi^{2}$ can have a Gaussian distribution by the central limit theorem, even when the noise in the original data is not Gaussian. For instance, if $n$ corresponds to the Poisson noise in the data with a small number of counts per bin, $\Delta \chi^{2}$ will have a Gaussian distribution provided that the tot number of counts contributing to $\sum \frac{\delta_{1,2} n}{\sigma^{2}}$ is large (Churazov et al. 1996). We can therefore use the original data with no grouping to calculate $\Delta \chi^{2}$.

\section{A.2. Dividing the Models Into a "More Plausible" Group and a "Less Plausible" Group}

For a given observed $\Delta \chi_{\mathrm{obs}}^{2}$ the ratio of likelihoods for two models is $\Lambda=\frac{L\left(M_{1}\right)}{L\left(M_{2}\right)}=e^{-\Delta \chi_{\mathrm{obs}}^{2} / 2}$, which corresponds to the Akaike information criterion (AIC) or Bayesian information criterion (BIC). Alternatively we can also employ the NeymanPearson lemma to differentiate between two models. If $M_{1}$ is the true model, then $M_{1}=M_{t}, \delta_{1}=0, \delta_{2}=-\delta_{1,2}$ and the
Table 8

Pairwise Comparison of Models from Table 2

\begin{tabular}{lcc}
\hline \hline Model & $\begin{array}{c}\text { ISGRI and SPI } \\
\text { (400-1350 keV) } \\
\text { Maximum Deviation }\end{array}$ & $\begin{array}{c}\text { ISGRI and SPI } \\
(70-1350 \mathrm{keV}) \\
\text { Maximum Deviation }\end{array}$ \\
\hline No source & 9.0 & 11.1 \\
DDT1p1 & 0.7 & 1.8 \\
DDT1p4halo & 1.1 & 2.1 \\
DDTe & 2.2 & 3.6 \\
DETO & 12.5 & 12.6 \\
HED6 & 4.3 & 5.4 \\
W7 & 0.8 & 1.3 \\
ddt1p4 & 2.3 & 1.3 \\
DD4 & 1.6 & 1.0 \\
\hline
\end{tabular}

Note. The ISGRI and SPI data for the early and late periods are used jointly. Large deviations imply that the model is disfavored by the data. Using a threshold of two standard deviations, one can conclude that No source, DETO, HED6, and (marginally) DDTe models are disfavored by the data, while the other models are compatible with the data. Since the 3Dbbal model was designed to fit the INTEGRAL data in the early period, this model has been excluded from this test. For each model the maximum deviation $\left|p_{\mathrm{bf}} / \sigma_{p}\right|$ with respect to all other models is given.

distribution of $\Delta \chi^{2}=\chi_{1}^{2}-\chi_{2}^{2}$ is

$$
\Delta \chi^{2}=-\sum\left(\frac{\delta_{1,2}}{\sigma}\right)^{2}+X \sqrt{4 \sum \frac{\delta_{1,2}^{2}}{\sigma^{2}}} .
$$

Then the probability $\alpha$ of getting $\Delta \chi^{2}>y$ is

$$
\alpha=Q(x)
$$

where $Q(x)=1 / 2 \operatorname{erfc}(x / \sqrt{2}), x=\frac{A+y}{2 \sqrt{A}}$, and $A=\sum\left(\frac{\delta_{1,2}}{\sigma}\right)^{2}$. Although the value of $A$ is known, we can take a conservative approach and write that $\frac{A+y}{2 \sqrt{A}} \geqslant \sqrt{y}$ for any $A \in[0, \infty]$. This value is achieved at $A=y$. Thus one can conclude that the conservative estimate of the probability of getting better $\chi^{2}$ for the wrong model (i.e., $\Delta \chi_{\mathrm{obs}}^{2}>y$ ) corresponds to more than $\sqrt{y}$ standard deviations. In the paper we use the value of $\Delta \chi_{\text {obs }}^{2}<4$ to separate the models into a "more plausible" group and a "less plausible". As we emphasized above, one can also interpret this value in the framework of BIC or AIC approaches.

\section{A.3. Pairwise Model Comparison and the Goodness-of-fit Criterion}

When comparing two models in terms of $\chi^{2}$ we are effectively projecting the $N$-dimensional data $D$ on a line connecting the models $M_{1}$ and $M_{2}$. We can formally introduce a linear parameter $p$, make a composite model $M_{1}+p\left(M_{2}-M_{1}\right)$, and calculate the best-fitting value $p_{\text {bf }}$ that minimizes $\chi^{2}$ :

$$
p_{\mathrm{bf}}=\frac{\sum-\left(D-M_{1}\right) \delta_{1,2} / \sigma^{2}}{\sum \delta_{1,2}^{2} / \sigma^{2}} .
$$

It is obvious that this quantity has a Gaussian distribution with the standard deviation

$$
\sigma_{p}=\left(\sum \delta_{1,2}^{2} / \sigma^{2}\right)^{-1 / 2} .
$$


We can now use the deviation of $p_{\mathrm{bf}}$ from 0 or 1 as a goodnessof-fit criterion for models $M_{1}$ and $M_{2}$, respectively. A significant deviation (i.e., $\left|p_{\mathrm{bf}} / \sigma_{p}\right| \gg 1$ or $\left.\left|\left(p_{\mathrm{bf}}-1\right) / \sigma_{p}\right| \gg 1\right)$ implies that one of the models (or both) is unlikely. While the goodness of fit can be calculated for each model alone, the power of the criterion depends on the data grouping (imagine, for example, a very weak signal distributed over a large number of bins). The goodnessof-fit calculation described above verifies only the projection of the data on the line set by models, but it "optimally" compares the difference between plausible models with the signal present in the data.

For each model used in Section 4 we have calculated the maximum deviation $\left|p_{\mathrm{bf}} / \sigma_{p}\right|$ with respect to all other models (except for the $3 \mathrm{Dbbal}$ model that was designed to fit the INTEGRAL data). Table 8 provides corresponding values when ISGRI and SPI data for the early and late periods are considered jointly. Based on this analysis we conclude that the No source, DETO, HED6, and (marginally) DDTe models are disfavored by the data, while the other models are compatible with the data.

\section{REFERENCES}

Amanullah, R., Goobar, A., Johansson, J., et al. 2014, ApJL, 788, L21 Ambwani, K., \& Sutherland, P. 1988, ApJ, 325, 820

Ashall, C., Mazzali, P., Bersier, D., et al. 2014, MNRAS, 445, 4427

Bachetti, M., Harrison, F. A., Walton, D. J., et al. 2014, Natur, 514, 202

Badenes, C., Bravo, E., Borkowski, K. J., \& Domínguez, I. 2003, ApJ, 593, 358

Bikmaev, I., Chugai, N., Sunyaev, R., et al. 2015, AstL, in press

Brown, P. J., Smitka, M. T., Wang, L., et al. 2015, ApJ, 805, 74

Churazov, E., Gilfanov, M., Forman, W., \& Jones, C. 1996, ApJ, 471, 673

Churazov, E., Sazonov, S., Tsygankov, S., Sunyaev, R., \& Varshalovich, D. 2011, MNRAS, 411, 1727

Churazov, E., Sunyaev, R., Grebenev, S., et al. 2014a, ATel, 5992, 1

Churazov, E., Sunyaev, R., Isern, J., et al. 2014b, Natur, 512, 406

Churazov, E., Sunyaev, R., Sazonov, S., Revnivtsev, M., \& Varshalovich, D. 2005, MNRAS, 357, 1377

Clayton, D. D., Colgate, S. A., \& Fishman, G. J. 1969, ApJ, 155, 75

Diehl, R., Siegert, T., Hillebrandt, W., et al. 2014, Sci, 345, 1162

Diehl, R., Siegert, T., Hillebrandt, W., et al. 2015, A\&A, 574, A72

Dong, S., Katz, B., Kushnir, D., \& Prieto, J. L. 2015, MNRAS, in press (arXiv:1401.3347)

Dotani, T., Hayashida, K., Inoue, H., Itoh, M., \& Koyama, K. 1987, Natur, 330,230

Fink, M., Kromer, M., Seitenzahl, I. R., et al. 2014, MNRAS, 438, 1762

Foley, R. J., Fox, O. D., McCully, C., et al. 2014, MNRAS, 443, 2887

Fossey, J., Cooke, B., Pollack, G., Wilde, M., \& Wright, T. 2014, CBET, 3792, 1

Friesen, B., Baron, E., Wisniewski, J. P., et al. 2014, ApJ, 792, 120

Goobar, A., Johansson, J., Amanullah, R., et al. 2014, ApJL, 784, L12

Goobar, A., Kromer, M., Siverd, R., et al. 2015, ApJ, 799, 106

Hillebrandt, W., \& Niemeyer, J. C. 2000, ARA\&A, 38, 191
Hoeflich, P., \& Khokhlov, A. 1996, ApJ, 457, 500

Hoyle, F., \& Fowler, W. A. 1960, ApJ, 132, 565

Iben, I., Jr., \& Tutukov, A. V. 1984, ApJS, 54, 335

Imshennik, V. S., \& Dunina-Barkovskaya, N. V. 2005, AstL, 31, 528

Isern, J., Jean, P., Bravo, E., et al. 2013, A\&A, 552, A97

Isern, J., Knoedlseder, J., Jean, P., et al. 2014, ATel, 6099, 1

Isern, J., Jean, P., Bravo, E., et al. 2015, A\&A, submitted

Jourdain, E., \& Roques, J. P. 2009, ApJ, 704, 17

Karachentsev, I. D., \& Kashibadze, O. G. 2006, Ap, 49, 3

Kawabata, K. S., Akitaya, H., Yamanaka, M., et al. 2014, ApJL, 795, LL4

Kelly, P. L., Fox, O. D., Filippenko, A. V., et al. 2014, ApJ, 790, 3

Kushnir, D., Katz, B., Dong, S., Livne, E., \& Fernández, R. 2013, ApJL, 778, LL37

Kuulkers, E. 2014, ATel, 5835, 1

Lebrun, F., Leray, J. P., Lavocat, P., et al. 2003, A\&A, 411, L141

Leloudas, G., Stritzinger, M. D., Sollerman, J., et al. 2009, A\&A, 505, 265

Maeda, K., Benetti, S., Stritzinger, M., et al. 2010, Natur, 466, 82

Malone, C. M., Nonaka, A., Woosley, S. E., et al. 2014, ApJ, 782, 11

Margutti, R., Parrent, J., Kamble, A., et al. 2014, ApJ, 790, 52

Marion, G. H., Sand, D. J., Hsiao, E. Y., et al. 2015, ApJ, 798, 39

Matz, S. M., Share, G. H., Leising, M. D., Chupp, E. L., \& Vestrand, W. T. 1988, Natur, 331, 416

Milne, P. A., Hungerford, A. L., Fryer, C. L., et al. 2004, ApJ, 613, 1101

Moll, R., Raskin, C., Kasen, D., \& Woosley, S. E. 2014, ApJ, 785, 105

Motohara, K., Maeda, K., Gerardy, C., et al. 2006, ApJL, 652, L101

Nadyozhin, D. K. 1994, ApJS, 92, 527

Nielsen, M. T. B., Gilfanov, M., Bogdán, Á, Woods, T. E., \& Nelemans, G. 2014, MNRAS, 442, 3400

Nomoto, K., \& Sugimoto, D. 1977, PASJ, 29, 765

Nomoto, K., Thielemann, F.-K., \& Yokoi, K. 1984, ApJ, 286, 644

Pakmor, R., Kromer, M., Taubenberger, S., et al. 2012, ApJL, 747, L10

Patat, F., Taubenberger, S., Cox, N. L. J., et al. 2015, A\&A, 577, A53

Pérez-Torres, M. A., Lundqvist, P., Beswick, R. J., et al. 2014, ApJ, 792, 38

Roques, J. P., Schanne, S., von Kienlin, A., et al. 2003, A\&A, 411, L91

Sazonov, S. Y., Lutovinov, A. A., \& Krivonos, R. A. 2014, AstL, 40, 65

Seitenzahl, I. R., Ciaraldi-Schoolmann, F., Röpke, F. K., et al. 2013, MNRAS, 429, 1156

Shigeyama, T., Nomoto, K., Yamaoka, H., \& Thielemann, F.-K. 1992, ApJL, 386, L13

Sturner, S. J., Shrader, C. R., Weidenspointner, G., et al. 2003, A\&A, 411, L81

Sunyaev, R., Kaniovsky, A., Efremov, V., et al. 1987, Natur, 330, 227

Sunyaev, R. A., Kaniovskii, A. S., Efremov, V. V., et al. 1990, SvAL, 16, 171

Teegarden, B. J., Barthelmy, S. D., Gehrels, N., Tueller, J., \& Leventhal, M. 1989, Natur, 339, 122

Telesco, C. M., Höflich, P., Li, D., et al. 2015, ApJ, 798, 93

The, L.-S., \& Burrows, A. 2014, ApJ, 786, 141

Ubertini, P., Lebrun, F., Di Cocco, G., et al. 2003, A\&A, 411, L131

Vedrenne, G., Roques, J.-P., Schönfelder, V., et al. 2003, A\&A, 411, L63

Webbink, R. F. 1984, ApJ, 277, 355

Welty, D. E., Ritchey, A. M., Dahlstrom, J. A., \& York, D. G. 2014, ApJ, 792, 106

Whelan, J., \& Iben, I., Jr. 1973, ApJ, 186, 1007

Winkler, C., Courvoisier, T. J.-L., di Cocco, G., et al. 2003, A\&A, 411, L1

Woosley, S. E., Axelrod, T. S., \& Weaver, T. A. 1981, CoNPP, 9, 185

Woosley, S. E., \& Weaver, T. A. 1991, in Supernovae, ed. J. Audouze et al. (Amsterdam: Elsevier), 63

Zheng, W., Shivvers, I., Filippenko, A. V., et al. 2014, ApJL, 783, L24 